\title{
Computing the topology of a real algebraic plane curve whose defining equations are available only "by values"
}

\author{
Robert M. Corless ${ }^{\mathrm{a}}$, Gema M. Diaz-Toca ${ }^{\mathrm{b}}$, Mario Fioravanti $^{\mathrm{c}, *}$, Laureano Gonzalez-Vega $^{\mathrm{c}}$, Ignacio F. Rua ${ }^{\mathrm{d}}$, \\ Azar Shakoori ${ }^{\mathrm{C}}$ \\ ${ }^{a}$ Department of Applied Mathematics, The University of Western Ontario, ON, Canada \\ ${ }^{b}$ Departamento de Matemática Aplicada, Universidad de Murcia, 30100 Murcia, Spain \\ ${ }^{c}$ Departamento de Matemáticas, Est. y Comp., Universidad de Cantabria, 39005 Santander, Spain \\ ${ }^{d}$ Departamento de Matemáticas, Universidad de Oviedo, 33007 Oviedo, Spain
}

\begin{abstract}
This paper is devoted to introducing a new approach for computing the topology of a real algebraic plane curve presented either parametrically or defined by its implicit equation when the corresponding polynomials which describe the curve are known only "by values". This approach is based on the replacement of the usual algebraic manipulation of the polynomials (and their roots) appearing in the topology determination of the given curve with the computation of numerical matrices (and their eigenvalues). Such numerical matrices arise from a typical construction in Elimination Theory known as the Bézout matrix which in our case is specified by the values of the defining polynomial equations on several sample points.
\end{abstract}

Keywords: Computations in the Lagrange Basis, Algebraic curve topology, Parametric curve topology, Generalized eigenvalues.

\section{Introduction}

The problem of computing the graph (even topologically) of an implicitly defined algebraic plane curve has received special attention from both Computer Aided Geometric Design and Symbolic Computation, independently. For the Computer Aided Geometric Design community, this problem is a basic subproblem appearing often in practice (see, for example, Bajaj et al. (1988), Keyser et al. (2000), Song et al. (2004), Farouki et al. (2005) or Hass et al. (2007)). For the Symbolic Computation community, on the other hand, this problem has been the motivation for many achievements in the study of subresultants, symbolic real root counting, infinitesimal computations, etc. By a comparison between the seminal papers Arnon and McCallum (1988), Gianni and Traverso (1983), Roy (1996), Sakkalis (1991), Cellini et al. (1991), Cucker et al. (1991), Feng (1992) and Roy and Szpirglas (1990) and the more renewed works of Gonzalez-Vega and El Kahoui (1996), Hong (1996), Gonzalez-Vega and Necula (2002), Busé et al. (2005), Seidel and Wolpert (2005), Eigenwillig et al. (July 2007), Lionel et al. (2008), Kerber (2009), Alcázar and Díaz-Toca (2010) and Cheng et al. (2010), one can see how the theoretical and practical complexities of the algorithms dealing with this problem have been dramatically improved.

This paper is devoted to introducing a new family of methods for determining the topology of a real algebraic plane curve presented either parametrically, $(x(t), y(t))$, or defined by its implicit equation $f(x, y)=0$. The main difference between our approach and those previously mentioned is the fact that here we completely replace the algebraic manipulation of the polynomials (and their roots) with computations of numerical matrices (and their eigenvalues). This is motivated by the fact that we prefer to deal with numerical matrices of moderate size instead of high degree polynomials. This is typically a better strategy from the numerical point of view when

\footnotetext{
* Corresponding author

Email addresses: rcorless@uwo.ca (Robert M. Corless), gemadiaz@um.es (Gema M. Diaz-Toca), mario.fioravanti@unican.es (Mario Fioravanti), laureano.gonzalez@unican.es (Laureano Gonzalez-Vega), rua@uniovi.es (Ignacio F. Rua), azar.shakoori@gmail.com (Azar Shakoori)
} 
the coefficients of the polynomials in the parametrization $(x(t), y(t))$ or of the implicit equation $f(x, y)=0$ are floating point real numbers.

But there is a stronger motivation behind the new approach to be introduced here. In many practical situations the polynomial $f(x, y)$ or the polynomials in $(x(t), y(t))$ appear presented in a non-expanded form which if expanded in the standard monomial basis produce polynomials of high degree, with big coefficients whose manipulation is a difficult task. For example, when computing the intersection of two surfaces, or when computing offset curves (for more examples see Diaz-Toca et al. (2012)). Otherwise, if the polynomials describing the considered curve are presented by their values then they can be easily evaluated at any desired point with a reasonable computational cost. This is our starting point: we do not assume that we have an explicit description (in the standard monomial basis) of the polynomial $f(x, y)$ or of the polynomials in $(x(t), y(t))$. We do only require, first, the knowledge of an upper bound for the degree of the polynomials which describe the considered real algebraic plane curve and second, the mathematical tools to evaluate those polynomials at any desired point.

Another application of the approach presented in this paper is to generate the points of an offset curve which is at distance $\delta$ from a parametric curve $(x(t), y(t))$ described by its values. Typically, the implicit equation of such an offset curve is either complicated to compute or is a dense bivariate polynomial of big size. A point $(x, y)$ is on the offset if, for some $t$, the following equations are verified:

$$
\begin{aligned}
& F(x, y ; t) \equiv(x-x(t))^{2}+(y-y(t))^{2}-\delta^{2}=0 \\
& G(x, y ; t) \equiv x^{\prime}(t)(x-x(t))+y^{\prime}(t)(y-y(t))=0 .
\end{aligned}
$$

The implicit equation can be obtained as the determinant of the Bézout matrix, $\operatorname{Bez}(F, G)$, with respect to $t$ (for a definition, see Section 1). For example, if we let the distance $\delta=1$, and the initial curve is parametrized by

$$
x(t)=t^{5}+t^{2}-3 t, \quad y(t)=\frac{t^{3}}{4}-t^{2}+1,
$$

the determinant of $\operatorname{Bez}(F, G)$ is a polynomial of degrees 14 and 18, in $x$ and $y$, respectively, with 158 terms whose coefficients have a number of significant digits ranging from 33 to 44 .

The paper is organized as follows: In Section 1, we detail some standard facts about the Bezoutian which is a tool from Elimination Theory. In Section 2, we introduce the linearization tools which are going to be used to determine the roots of the determinant of a matrix polynomial without computing the determinant. In other words, instead of computing the determinant and then its roots we compute the so-called generalized eigenvalues of a matrix pencil (in this context known as the companion matrix pencil). In Section 3, we give an explicit description of how to compute the Bézout matrix for a pair of univariate polynomials and the companion matrix pencil associated with a matrix polynomial, in the Lagrange polynomial basis. In Section 4, we summarize the standard strategies used for computing the topology of $f(x, y)=0$; we show how algebraic manipulation of polynomials can be replaced with the computation of the Bézout matrix by values and the corresponding generalized eigenvalues. Section 5 and Section 6 are devoted to introducing the algorithms for computing the topology of a real algebraic plane curve presented by its implicit equation $f(x, y)=0$ or by its parametrization $(x(t), y(t))$ in the Lagrange polynomial basis. Both sections include a detailed description of the corresponding algorithm together with several examples and the results coming from our tests and experimentations. Section 7 shows how to compute the topology of a real algebraic plane curve presented by its parametrization $(x(t), y(t))$ in the Lagrange polynomial basis by applying the same algorithm used for the implicit equation case in Section 5; in this case it is enough to show how to evaluate the implicit equation $f(x, y)$ of $(x(t), y(t))$ without computing $f(x, y)$, explicitly. In Section 8 , we briefly discuss some numerical issues posed by our algorithms. Finally, in Section 9, we summarize the results and highlight several points where we believe our approach can be improved.

\section{Basic notation}

In this paper, vectors and matrices are denoted by bold letters. The notation $p(t) \in \mathbb{K}[t]$ indicates a univariate polynomial in the variable $t$ with coefficients from $\mathbb{K}$. We denote the vector space of the polynomials of degree at most $n$ by $\mathbb{P}_{n}$. For bivariate polynomials of degree at most $m$ in the first argument and degree at most $n$ in the second argument, the associated vector space is denoted $\mathbb{P}_{m, n}$. For a bivariate polynomial $p(z, t)$, 
$\operatorname{deg}_{z}(p(z, t))\left(\operatorname{resp} \cdot \operatorname{deg}_{t}(p(z, t))\right)$ denotes the degree of $p$ when considered as a polynomial in $z$ (resp. $t$ ) with polynomial coefficients in $t$ (resp. $z$ ). The space of $n \times n$ square matrices with elements in $\mathbb{K}$ will be denoted by $\mathcal{M}_{n}(\mathbb{K})$.

\section{The Bezoutian in the monomial basis}

Standard definitions of the Bezoutian make explicit reference to the monomial or power basis (see, e.g., Barnett (1990)). However, all constructions of the Bézout matrix make use of the so-called Cayley quotient which makes no reference to any particular basis in which the given polynomials are represented.

Definition 1. Let $p(t), q(t)$ be univariate polynomials with $n=\max \{\operatorname{deg}(p(t)), \operatorname{deg}(q(t))\}$. The Cayley quotient of $p(t)$ and $q(t)$ is the polynomial $C_{p, q}$ of degree at most $n-1$ defined by

$$
C_{p, q}(z, t)=\frac{p(t) q(z)-p(z) q(t)}{t-z}
$$

Although $C_{p, q}$ as written in (2) is a rational function of $z$ and $t$, the numerator vanishes if $z=t$; as such $t-z$ divides $p(t) q(z)-p(z) q(t)$, and the Cayley quotient $C_{p, q}$ is a bivariate polynomial in $\mathbb{P}_{n-1, n-1}$, as stated. The apparent discontinuity in the Cayley quotient $C_{p, q}(z, t)$ in equation (2) when $t$ goes to $z$ can be removed (see Shakoori (2007) for a proof):

$$
\lim _{t \rightarrow z} C_{p, q}(z, t)=p^{\prime}(z) q(z)-p(z) q^{\prime}(z)
$$

Definition 2. If $\boldsymbol{\Phi}(t)=\left[\phi_{1}(t), \ldots, \phi_{n}(t)\right]$ is a basis for $\mathbb{P}_{n-1}$ then $C_{p, q}$ in (2) can be uniquely written

$$
C_{p, q}(z, t)=\sum_{i, j=1}^{n} b_{i j} \phi_{i}(z) \phi_{j}(t) .
$$

The symmetric matrix with coefficients $b_{i j}$ is called the Bezoutian or the Bézout matrix associated with $p(t)$ and $q(t)$ in the polynomial basis $\boldsymbol{\Phi}$.

Note that if $t^{*} \in \mathbb{C}$ is a common zero of $p(t)$ and $q(t)$, then $\boldsymbol{\Phi}\left(t^{*}\right)$ is a vector in the nullspace of $\mathbf{B}$.

The construction of the Bézout matrix using the Cayley quotient in the monomial basis (i.e., power basis) is classic (see for example Barnett (1990)). The computation of the Bézout matrix in the Bernstein and Chebyshev bases have been studied in Bini and Gemignani (2004) and Barnett (1989), respectively.

Definition 3. Let $p(t)$ and $q(t)$ be as described in Definition 1. The Bézout matrix associated with $p(t)$ and $q(t)$, in the monomial basis, is the symmetric matrix:

$$
\operatorname{Bez}(p, q)=\left(\begin{array}{ccc}
b_{0,0} & \ldots & b_{0, n-1} \\
\vdots & & \vdots \\
b_{n-1,0} & \ldots & b_{n-1, n-1}
\end{array}\right)
$$

where the elements $b_{i, j}$ are defined by the Cayley quotient:

$$
\frac{p(t) q(z)-p(z) q(t)}{t-z}=\sum_{i, j=0}^{n-1} b_{i, j} t^{i} z^{j}
$$

The Bezoutian determines the degree of the greatest common divisor of $p$ and $q \operatorname{because} \operatorname{deg}(\operatorname{gcd}(p, q))=$ $n-\operatorname{rank}(\operatorname{Bez}(p, q))$. The rank of $\operatorname{Bez}(p, q)$ is equal to the order of the largest nonsingular principal minor, when starting from the lower right hand corner (see Bini and Pan (1994) and Helmke and Fuhrmann (1989)).

The nullspace of the Bezoutian has an elegant structure presented in the next proposition that can be used to determine the common roots of the given polynomials (for a proof see Heinig and Rost (1984), page 42). 
Proposition 1. The nullspace of $\operatorname{Bez}(p, q)$ is spanned by

$$
\operatorname{nullspace}(\operatorname{Bez}(p, q))=\left[X_{1}, X_{2}, \ldots, X_{k}\right]
$$

where each block $X_{j}$ corresponds to a different common root of $p$ and $q$. The dimension of each block is the geometric multiplicity $k_{j}$ of the common root $x_{j}$ (i.e., its multiplicity as a root of the greatest common divisor of $p$ and $q)$. Moreover each block can be parameterized by the common root $x_{j}$ in the form

$$
X_{j}=\left(\begin{array}{ccccc}
1 & 0 & 0 & \cdots & 0 \\
x_{j} & 1 & 0 & & 0 \\
x_{j}^{2} & 2 x_{j} & 2 & & \vdots \\
x_{j}^{3} & 3 x_{j}^{2} & 6 x_{j} & \ddots & \vdots \\
\vdots & \vdots & \vdots & \ddots & \left(k_{j}-1\right) ! \\
\vdots & \vdots & \vdots & & k_{j} ! x_{j} \\
\vdots & \vdots & & & \vdots \\
x_{j}^{n-1} & (n-1) x_{j}^{n-2} & (n-1)(n-2) x_{j}^{n-3} & \ldots & (n-1) \frac{k_{j}-1}{x_{j}^{n-k_{j}}}
\end{array}\right)
$$

where $n \underline{k_{j}}=n(n-1) \cdots\left(n-k_{j}+1\right)$.

In particular, when the nullspace is of dimension 1 , it is generated by $\left[1, x, x^{2}, \ldots, x^{n-1}\right]^{T}$ for $x$ the unique common root of $p(t)$ and $q(t)$. We may, if we choose, order the construction of the quadratic form for the Bezoutian in such a way as to reverse the row order of the entries of the nullspace.

Finally the next proposition and corollary show how the structure of the nullspace for the Bezoutian can be very helpful to deal with the computation of multiple roots (under certain additional hypothesis).

Proposition 2. Let $p$ be a polynomial in $\mathbb{C}[t]$. If $i \geq 1, p^{(i)}=\frac{\mathrm{d}^{i} p}{\mathrm{~d} t^{i}}$ and $N_{i}$ is the subspace defined by:

$$
N_{i}=\bigcap_{j=1}^{i} \operatorname{nullspace}\left(\operatorname{Bez}\left(p, p^{(j)}\right)\right)
$$

then

$$
\operatorname{dim}\left(N_{i}\right)=\operatorname{deg}\left(\operatorname{gcd}\left(p, p^{(1)}, \ldots, p^{(i)}\right)\right) .
$$

Proof. If $n=\operatorname{deg}(p)$ and $\Theta$ is the linear mapping from $\mathbb{C}^{n}$ to $\mathbb{C}^{n i}$ given by the matrix

$$
\left(\begin{array}{c}
\operatorname{Bez}\left(p, p^{(1)}\right) \\
\operatorname{Bez}\left(p, p^{(2)}\right) \\
\vdots \\
\operatorname{Bez}\left(p, p^{(i)}\right)
\end{array}\right)
$$

(columnwise) then

$$
\operatorname{nullspace}(\Theta)=\bigcap_{j=1}^{i} \operatorname{nullspace}\left(\operatorname{Bez}\left(p, p^{(j)}\right)\right)=N_{i} \text {. }
$$

By applying Theorem 3.2 in Diaz-Toca and Gonzalez-Vega (2002):

$$
\begin{aligned}
\operatorname{dim}\left(N_{i}\right) & =\operatorname{dim}(\operatorname{nullspace}(\Theta))=n-\operatorname{rank}\left(\begin{array}{c}
\operatorname{Bez}\left(p, p^{(1)}\right) \\
\operatorname{Bez}\left(p, p^{(2)}\right) \\
\vdots \\
\operatorname{Bez}\left(p, p^{(i)}\right)
\end{array}\right) \\
& =n-\left(n-\operatorname{deg}\left(\operatorname{gcd}\left(p, p^{(1)}, \ldots, p^{(i)}\right)\right)\right)=\operatorname{deg}\left(\operatorname{gcd}\left(p, p^{(1)}, \ldots, p^{(i)}\right)\right)
\end{aligned}
$$

as desired. 
Corollary 1. A polynomial $p \in \mathbb{C}[t]$ has exactly one multiple root of multiplicity $m+1$ (and no other multiple roots) if and only if $\operatorname{dim}\left(N_{i}\right)-\operatorname{dim}\left(N_{i+1}\right)=1$ for $i$ in $\{1, \ldots, m\} \quad$ (note that this implies that $N_{m+1}=\{\mathbf{0}\}$ ). Moreover, if $\alpha$ is such a unique multiple root of multiplicity $m+1$ and $\left(v_{1}, \ldots, v_{n}\right)$ is any nonzero vector in $N_{m}$, then

$$
\alpha=\frac{v_{2}}{v_{1}} .
$$

\section{Generalized eigenvalue problems}

The classical discriminant computation for determining the $x$-projection of the critical points of the real algebraic plane curve defined by the equation $f(x, y)=0$ is going to be replaced by its formulation as a nonlinear eigenvalue problem. In other words, instead of computing the real roots of the determinant of a matrix polynomial directly, we solve a sparse generalized eigenvalue problem for a regular matrix pencil.

In this section we review some of the main properties of generalized eigenvalues to be used in the next sections. See Amiraslani et al. (2009); Corless et al. (2007) for a more detailed discussion.

We define an $n \times n$ matrix polynomial as an $n \times n$ matrix $\mathbf{A}(t)$ whose entries $A_{i j}(t)$ are polynomials in $t$ $(1 \leq i, j \leq n)$. The degree of a matrix polynomial $\mathbf{A}(t)$ is the maximum degree of its entries, i.e.,

$$
\operatorname{deg}(\mathbf{A}(t))=\max _{1 \leq i, j \leq n} \operatorname{deg}\left(A_{i j}(t)\right) .
$$

Given a matrix polynomial $\mathbf{A}(t)$, the polynomial eigenvalue problem is as follows:

Find $t^{*} \in \mathbb{C}$ such that $\mathbf{A}\left(t^{*}\right)$ is singular.

There is an extensive literature on such problems; theoretical results about solutions of polynomial eigenvalue problems are available in Gohberg et al. (1982); Manocha and Demmel (1995). Moreover, standard algorithms exist for polynomial eigenvalue problems expressed in the power basis through linearization, i.e., finding an associated pair of companion matrices that defines a generalized eigenvalue problem. The finite generalized eigenvalues of such a pair are exactly the polynomial eigenvalues of the matrix polynomial $\mathbf{A}(t)$ (see Demmel (1997); Manocha and Demmel (1995); Tisseur and Higham (2001) and, e.g., Matlab's polyeig function).

If

$$
\mathbf{A}(t)=\sum_{i=0}^{m} \mathbf{A}_{i} t^{i},
$$

where each $\mathbf{A}_{i}$ is a numerical square matrix of size $n$, then we construct (with $\mathbf{I}$ and $\mathbf{0}$ denoting the identity and the zero matrices of size $n$, respectively)

$$
\mathbf{C}_{0}=\left(\begin{array}{ccccc}
\mathbf{0} & \mathbf{0} & \ldots & \mathbf{0} & -\mathbf{A}_{0} \\
\mathbf{I} & \mathbf{0} & \ldots & \mathbf{0} & -\mathbf{A}_{1} \\
\mathbf{0} & \mathbf{I} & \ldots & \mathbf{0} & -\mathbf{A}_{2} \\
\vdots & \vdots & & \vdots & \vdots \\
\mathbf{0} & \mathbf{0} & \ldots & \mathbf{0} & -\mathbf{A}_{m-2} \\
\mathbf{0} & \mathbf{0} & \ldots & \mathbf{I} & -\mathbf{A}_{m-1}
\end{array}\right), \quad \mathbf{C}_{1}=\left(\begin{array}{ccccc}
\mathbf{I} & \mathbf{0} & \ldots & \mathbf{0} & \mathbf{0} \\
\mathbf{0} & \mathbf{I} & \ldots & \mathbf{0} & \mathbf{0} \\
\vdots & \vdots & & \vdots & \vdots \\
\vdots & \vdots & & \vdots & \vdots \\
\mathbf{0} & \mathbf{0} & \ldots & \mathbf{I} & \mathbf{0} \\
\mathbf{0} & \mathbf{0} & \ldots & \mathbf{0} & \mathbf{A}_{m}
\end{array}\right)
$$

It can be verified that $\operatorname{det}\left(t \mathbf{C}_{1}-\mathbf{C}_{0}\right)=\operatorname{det}(\mathbf{A}(t))$. The pair of matrices $\left(\mathbf{C}_{0}, \mathbf{C}_{1}\right)$ is called a companion matrix pencil of $\mathbf{A}(t)$, and $t \in \mathbb{C}$ such that $\operatorname{det}\left(t \mathbf{C}_{1}-\mathbf{C}_{0}\right)=0$ is called a generalized eigenvalue. Similar to an ordinary eigenvalue problem, for a given generalized eigenvalue, we have left and right eigenvectors. The matrix pencil is called regular if $\operatorname{det}\left(t \mathbf{C}_{1}-\mathbf{C}_{0}\right)$ is not identically zero.

Usually the degree of $\operatorname{det}\left(t \mathbf{C}_{1}-\mathbf{C}_{0}\right)$ is smaller than $n m$ : in that case we say that $\infty$ is a generalized eigenvalue for the matrix pencil $\left(\mathbf{C}_{0}, \mathbf{C}_{1}\right)$ with multiplicity $n m-\operatorname{deg}\left(\operatorname{det}\left(t \mathbf{C}_{1}-\mathbf{C}_{0}\right)\right)$. The next proposition gives a more concrete description of the multiplicity of $\infty$ as a generalized eigenvalue for $\left(\mathbf{C}_{0}, \mathbf{C}_{1}\right)$ (see Amiraslani et al. (2009)).

Proposition 3. The multiplicity of $\infty$ as a generalized eigenvalue for the matrix pencil $\left(\mathbf{C}_{0}, \mathbf{C}_{1}\right)$ is at least $n-\operatorname{rank}\left(\mathbf{A}_{m}\right)$, and it is equal to the multiplicity of 0 as a generalized eigenvalue of the matrix pencil $\left(\mathbf{C}_{1}, \mathbf{C}_{0}\right)$. 


\section{The Bézout matrix in the Lagrange basis}

There are several applications of Bézout matrices for bivariate polynomials given by samples in each variable. The natural basis is the tensor product of the Lagrange basis in each variable. Before discussing such applications we review some theories and facts about the Lagrange interpolation problem (for the proofs and a more detailed discussion see Shakoori (2007); Corless (2004)). We consider the so-called barycentric representation of the Lagrange basis which is known to have numerical advantages over the familiar standard definition (see Berrut and Trefethen (2004); Schneider and Werner (1984) and Section 8 below).

Definition 4. Let $\boldsymbol{\tau}=\left(\tau_{1}, \ldots, \tau_{d+1}\right) \in \mathbb{C}^{d+1}$ be a vector whose numerical entries are all distinct. We define

$$
\ell(t ; \boldsymbol{\tau})=\prod_{j=1}^{d+1}\left(t-\tau_{j}\right) \in \mathbb{P}_{d}, \quad \omega_{i}(\boldsymbol{\tau})=\left[\prod_{\substack{j=1 \\ j \neq i}}^{d+1}\left(\tau_{i}-\tau_{j}\right)\right]^{(-1)},
$$

where $\omega_{i}(\boldsymbol{\tau})$ are the barycentric weights. The associated barycentric Lagrange polynomials are given by

$$
L_{i}(t ; \boldsymbol{\tau})=\frac{\omega_{i}(\boldsymbol{\tau}) \ell(t ; \boldsymbol{\tau})}{t-\tau_{i}}, \quad(1 \leq i \leq d+1),
$$

If $p(t) \in \mathbb{P}_{d}$ and $p_{i}=p\left(\tau_{i}\right), i=1, \ldots, d+1$, then

$$
p(t)=\ell(t ; \boldsymbol{\tau}) \sum_{i=1}^{d+1} \frac{\omega_{i}(\boldsymbol{\tau}) p_{i}}{t-\tau_{i}} .
$$

Moreover, we call

$$
\mathbf{L}(t ; \boldsymbol{\tau})=\left[L_{1}(t ; \boldsymbol{\tau}), \ldots, L_{d+1}(t ; \boldsymbol{\tau})\right]^{T} \in\left[\mathbb{P}_{d}\right]^{d+1},
$$

the Lagrange interpolation basis.

Dealing with some geometric problems involving plane curves (such as those considered in this paper) requires the computation of the Bézout matrix that is in fact a matrix polynomial which in turn gives rise to a polynomial eigenvalue problem described in Section 2.

Proposition 4. Let $p(t), q(t) \in \mathbb{P}_{d}$ with $d=\max \{\operatorname{deg}(p(t)), \operatorname{deg}(q(t))\}$. Suppose $\boldsymbol{\tau}=\left(\tau_{1}, \ldots, \tau_{d+1}\right) \in \mathbb{C}^{d+1}$ consists of distinct numerical values. Let $\boldsymbol{p}=\left(p_{1}, \ldots, p_{d+1}\right)$ and $\boldsymbol{q}=\left(q_{1}, \ldots, q_{d+1}\right)$ be numerical data such that

$$
p\left(\tau_{i}\right)=p_{i} \quad \text { and } \quad q\left(\tau_{i}\right)=q_{i} \quad(1 \leqslant i \leqslant d+1) .
$$

Let $\tilde{\boldsymbol{\tau}}=\left(\tau_{1}, \ldots, \tau_{d}\right) \in \mathbb{C}^{d}$ consist of all the nodes in $\boldsymbol{\tau}$ except $\tau_{d+1}$. Let $p_{i}^{\prime}=p^{\prime}\left(\tau_{i}\right)$ and $q_{i}^{\prime}=q^{\prime}\left(\tau_{i}\right)$ denote the values of the derivatives of $p(t)$ and $q(t)(1 \leqslant i \leqslant d)$. Then, the Bézout matrix in the Lagrange basis $\mathbf{L}(t ; \tilde{\boldsymbol{\tau}}) \in\left[\mathbb{P}_{d-1}\right]^{d}$ is the matrix $\operatorname{Bez}(p, q)=\left[b_{i, j}\right]$ with entries given by

$$
\begin{aligned}
& b_{i, j}=\frac{p_{i} q_{j}-p_{j} q_{i}}{\tau_{i}-\tau_{j}}, \quad i \neq j \\
& b_{i, i}=p_{i}^{\prime} q_{i}-p_{i} q_{i}^{\prime}, \quad i=j
\end{aligned}
$$

In order to avoid a cumbersome notation, we use the same notation $\operatorname{Bez}(p, q)$ as in the monomial basis. In the cases where there might be some doubt, the used basis will be mentioned.

Lemma 1 (Matrix congruence property). Let $\boldsymbol{\tau}, p(t), q(t) \in \mathbb{P}_{d}$, and $\tilde{\boldsymbol{\tau}}$ be as defined in Proposition 4. Moreover, let $\mathbf{V} \in \mathbb{C}^{d \times d}$ be the Vandermonde matrix associated with $\tilde{\boldsymbol{\tau}}$.

$$
\mathbf{V}=\left(\begin{array}{cccc}
1 & \tau_{1} & \ldots & \tau_{1}^{d-1} \\
1 & \tau_{2} & \ldots & \tau_{2}^{d-1} \\
\vdots & \vdots & \vdots & \vdots \\
1 & \tau_{d} & \ldots & \tau_{d}^{d-1}
\end{array}\right)
$$


Let also $\mathbf{B}$ denote $\operatorname{Bez}(p, q)$ associated with $\tilde{\boldsymbol{\tau}}$, in the Lagrange basis, $\mathbf{L}(t ; \tilde{\boldsymbol{\tau}})$ and $\mathbf{B}_{M}$ the corresponding Bezoutian in the monomial basis. Then,

$$
\mathbf{B}=\mathbf{V} \mathbf{B}_{M} \mathbf{V}^{T} .
$$

As we showed in Proposition 4, to compute the diagonal entries of the Bézout matrix in the Lagrange basis, we need to calculate $p_{i}^{\prime}$ and $q_{i}^{\prime},(1 \leqslant i \leqslant d)$. In fact, we need to have a systematic way of computing all the derivatives of the polynomial interpolant on the given sample points. Next we introduce a formula which is the main tool in computing the diagonal entries of the Bézout matrix in the Lagrange basis.

Lemma 2. Let $\boldsymbol{\tau}=\left(\tau_{1}, \tau_{2}, \ldots, \tau_{d+1}\right) \in \mathbb{C}^{d+1}$ consist of distinct sample points, and $\mathbf{p}=\left(p_{1}, p_{2}, \ldots, p_{d+1}\right)$ be the numerical values of the polynomial $p(t) \in \mathbb{P}_{d}$ at $\boldsymbol{\tau}$, Moreover, let $\omega_{i}(\boldsymbol{\tau})$, and $\omega_{j}(\boldsymbol{\tau})$ be the barycentric weights constructed by using Equation (8). The values $p_{i}^{\prime}=p^{\prime}\left(\tau_{i}\right)$ of the derivative of the interpolant $p^{\prime}(t) \in \mathbb{P}_{d-1}$ at the nodes in $\boldsymbol{\tau}$ are

$$
p_{i}^{\prime}=\frac{1}{\omega_{i}(\boldsymbol{\tau})} \sum_{\substack{j=1 \\ j \neq i}}^{d+1} \frac{\omega_{j}(\boldsymbol{\tau})\left(p_{j}-p_{i}\right)}{\tau_{i}-\tau_{j}} \quad(1 \leq i \leq d+1) .
$$

Proof. See Amiraslani (2006); Trefethen (2000).

Observe that in this case, if $t^{*} \in \mathbb{C}$ is a common zero of $p(t)$ and $q(t)$, then $\mathbf{L}\left(t^{*} ; \tilde{\boldsymbol{\tau}}\right)$ is a null vector of the Bézout matrix with respect to the Lagrange basis.

Theorem 1. Let $p(t), q(t) \in \mathbb{P}_{d}$ with $d=\max (\operatorname{deg}(p(t)), \operatorname{deg}(q(t)))$. We assume that $p(t)$ and $q(t)$ are specified in the Lagrange basis, by data $\boldsymbol{\tau}, \mathbf{p}$, and $\mathbf{q}$ as in Proposition 4. If $p(t), q(t)$ has only one common root, $t^{*}$, which is simple, then we may use any vector $\boldsymbol{U}=\left[u_{1}, u_{2}, \ldots, u_{d}\right]^{T}$ in the nullspace of the corresponding Bézout matrix in the Lagrange basis to compute $t^{*}$. This is done by means of a procedure that is referred to as "taking moments". That is,

$$
t^{*}=\frac{\sum_{i=1}^{d} \tau_{i} u_{i}}{\sum_{i=1}^{d} u_{i}} .
$$

Proof. By hypothesis, for $1 \leqslant i \leqslant d$, we have $u_{i}=\alpha L_{i}\left(t^{*} ; \tilde{\boldsymbol{\tau}}\right)$ for some $\alpha$. Then

$$
\sum_{i=1}^{d} u_{i}=\alpha \sum_{i=1}^{d} L_{i}\left(t^{*} ; \tilde{\boldsymbol{\tau}}\right)=\alpha .
$$

This is because the sum interpolates 1 on $\tilde{\boldsymbol{\tau}}$. Likewise,

$$
\sum_{i=1}^{d} \tau_{i} u_{i}=\alpha \sum_{i=1}^{d} \tau_{i} L_{i}\left(t^{*} ; \tilde{\boldsymbol{\tau}}\right)=t^{*} \alpha
$$

because the sum interpolates $t$. By replacing the right hand sides of the Equations (18) and (19) in Equation (17), the proof is complete.

On the other hand, similar to the monomial basis case described in Section 2, for a matrix polynomial $\mathbf{A}(t)$ written in the Lagrange basis $\mathbf{L}(t ; \boldsymbol{\tau})$, we construct a block matrix pencil $\left(\mathbf{C}_{0}, \mathbf{C}_{1}\right)$ whose finite generalized eigenvalues include the roots of $\operatorname{det}(\mathbf{A}(t))$.

Definition 5. We consider the matrix polynomial

$$
\mathbf{A}(t)=\mathbf{A}_{1} L_{1}(t ; \boldsymbol{\tau})+\mathbf{A}_{2} L_{2}(t ; \boldsymbol{\tau})+\ldots+\mathbf{A}_{d+1} L_{d+1}(t ; \boldsymbol{\tau}) .
$$


where, for $1 \leqslant i \leqslant d+1, \mathbf{A}_{i}$ are $r \times r$ matrices such that $\mathbf{A}\left(\tau_{i}\right)=\mathbf{A}_{i}$. The corresponding companion matrix pencil for the matrix polynomial $\mathbf{A}(t)$ is

$$
\mathbf{C}_{0}, \mathbf{C}_{1}=\left(\begin{array}{ccccc}
\tau_{1} \mathbf{I} & & & & \mathbf{A}_{1} \\
& \tau_{2} \mathbf{I} & & & \mathbf{A}_{2} \\
& & \ddots & & \vdots \\
& & & \tau_{d+1} \mathbf{I} & \mathbf{A}_{d+1} \\
-\omega_{1} \mathbf{I} & -\omega_{2} \mathbf{I} & \cdots & -\omega_{d+1} \mathbf{I} & \mathbf{0}
\end{array}\right),\left(\begin{array}{ccccc}
\mathbf{I} & & & & \\
& \mathbf{I} & & & \\
& & \ddots & & \\
& & & \mathbf{I} & \\
& & & \mathbf{0}
\end{array}\right),
$$

where $\mathbf{I}$ and $\mathbf{0}$ are the identity matrix and the zero matrix, respectively, conformal with the $r \times r$ matrices $\mathbf{A}_{i}$, for $1 \leqslant i \leqslant d+1$. Matrices $\mathbf{C}_{0}$ and $\mathbf{C}_{1}$ are of dimension $r(d+2) \times r(d+2)$ and we have that $\operatorname{det} \mathbf{A}(t)=$ $\operatorname{det}\left(t \mathbf{C}_{1}-\mathbf{C}_{0}\right)$.

Although the purpose of the present paper is not to discuss methods for solving generalized eigenvalue problems, we will make some brief considerations in Section 8.

\section{Characterizing the topology of $f(x, y)=0$}

The goal of this section is to recall the main ideas of the usual method for computing the topology of algebraic curves, from which our method has, in some sense, evolved. This will make the exposition clearer, and the paper self-contained. The characterization of the topology of a curve $\mathcal{C}_{f}$ presented by the equation $f(x, y)=0$ is usually based on the location of the critical points of $f$ with respect to $y$ (i.e., singular points or points with a vertical tangent) and on the study of the halfbranches (i.e., simple arcs of curve starting from a point) of $\mathcal{C}_{f}$ around those points (see for example Hong (1996); Gonzalez-Vega and El Kahoui (1996); GonzalezVega and Necula (2002); Seidel and Wolpert (2005); Cheng et al. (2010)). This is due to the fact that for any other point of $\mathcal{C}_{f}$ there will be only one halfbranch to the left and one halfbranch to the right.

Definition 6. Let $f(x, y) \in \mathbb{R}[x, y]$ and $\mathcal{C}$ be the real algebraic plane curve defined by $f$ :

$$
\mathcal{C}_{f}=\left\{(\alpha, \beta) \in \mathbb{R}^{2}: f(\alpha, \beta)=0\right\} .
$$

$A$ point $(\alpha, \beta) \in \mathbb{C}^{2}$ is called a critical point of $\mathcal{C}_{f}$ if

$$
f(\alpha, \beta)=0, \frac{\partial f}{\partial y}(\alpha, \beta)=0 .
$$

$A$ critical point is said to be singular if $\frac{\partial f}{\partial x}(\alpha, \beta)=0$; nonsingular critical points are called ramification points. $A$ point $(\alpha, \beta) \in \mathbb{C}^{2}$ is a regular point of $\mathcal{C}_{f}$ if $f(\alpha, \beta)=0$ and it is not critical.

The usual strategy to compute the topological graph of a real algebraic plane curve $\mathcal{C}$ defined implicitly by a polynomial $f(x, y) \in \mathbb{R}[x, y]$ proceeds in the following way:

1. Compute the squarefree part of the discriminant of $f$ with respect to $y, D(x)$, and determine its real roots, $\alpha_{1}<\alpha_{2}<\ldots<\alpha_{r}$ which are the $x$-coordinates of the critical points of $\mathcal{C}_{f}$.

2. For every $\alpha_{i}$, compute the real roots of $f\left(\alpha_{i}, y\right), \beta_{i, 1}<\ldots<\beta_{i, s_{i}}$, determining which $\left(\alpha_{i}, \beta_{i, j}\right)$ is regular and which $\left(\alpha_{i}, \beta_{i, j}\right)$ is critical.

3. For every $\left(\alpha_{i}, \beta_{i, j}\right)$, compute the number of halfbranches to the right and to the left of the point $\left(\alpha_{i}, \beta_{i, j}\right)$.

Steps 1 and 2 provide the vertices of a graph representing the topology of $\mathcal{C}_{f}$ such as the one in Figure 3 . For the purpose of tracing the curve numerically, this graph is of much importance because it helps us understand how to proceed when we approach a complicated point on the curve.

In this section we summarize two usual different approaches for solving the steps 1 and 2 . The main difference between the two approaches is that in the method we choose in order to determine the $x$-coordinates of the critical points: we can either choose to explicitly compute the discriminant of $f(x, y)$ with respect to $y$, or we can avoid such computation. Although these two methods use the monomial expression of $f(x, y)$, their presentation here is necessary to understand the rationale behind the method presented in Section 5 in which we do not have the monomial expression of $f(x, y)$. 


\subsection{Using the discriminant}

We begin with the computation of the discriminant $R(x)$ of $f$ with respect to $y$ by any available method (a determinant computation, subresultant algorithm, etc.) and of its squarefree part:

$$
D(x)=\frac{R(x)}{\operatorname{gcd}\left(R(x), R^{\prime}(x)\right)} .
$$

This step ends with the computation of the real roots of $D(x)$.

When solving the equations $f\left(\alpha_{i}, y\right)=0$, to avoid the numerical problems arising from the computation of the multiple roots of every $f\left(\alpha_{i}, y\right)$, a linear change of coordinates might be necessary. Such change of coordinates puts the curve in a desirable position which is known as "generic position" (see Gonzalez-Vega and Necula (2002)).

Definition 7. Let $f \in \mathbb{R}[x, y]$ be a squarefree polynomial. The real algebraic plane curve defined by $f, \mathcal{C}_{f}$, is in generic position if the following two conditions are satisfied:

1. The leading coefficient of $f$ with respect to $y$ (which is a polynomial in $\mathbb{R}[x]$ ) has no real roots.

2. For every $\alpha \in \mathbb{R}$ the number of distinct complex roots of

$$
f(\alpha, y)=0, \quad \frac{\partial f}{\partial y}(\alpha, y)=0
$$

is 0 or 1 .

One of the advantages of having the curve in generic position is presented in the next proposition (for a more detailed discussion see Necula (2005); Gonzalez-Vega and Necula (2002)).

Proposition 5. Let $f \in \mathbb{R}[x, y]$ be a squarefree polynomial such that $\mathcal{C}_{f}$ is in generic position. If $(\alpha, \beta)$ is a critical point of $\mathcal{C}_{f}$ and $\alpha \in \mathbb{R}$ then $\beta \in \mathbb{R}$ too.

In general, if $(\alpha, \beta)$ is a critical point on $\mathcal{C}_{f}$ and $\alpha$ is a real number, this does not imply that $\beta$ is a real number as well (see example 1).

If $\mathcal{C}_{f}$ is in generic position then for every real root $\alpha$ of $D(x)$ there is only one critical point of the curve in the vertical line $x=\alpha$ whose $y$-coordinate can be rationally described in terms of $\alpha$; if the equation $f(x, y)$ is available as a polynomial in $y$ whose coefficients are polynomials in $x$ then, by using subresultants and for each critical point $(\alpha, \beta)$, a rational function $r_{\alpha}(x) \in \mathbb{R}(x)$ is explicitly determined such that

$$
\beta=r_{\alpha}(\alpha) .
$$

To check if $\mathcal{C}_{f}$ is in generic position the following condition is used (see Theorem 3.7 in Gonzalez-Vega and Necula (2002)):

$\mathcal{C}_{f}$ is in generic position if and only if for each $\alpha, \operatorname{Sres}_{k_{i}}\left(f(\alpha, y), f_{y}(\alpha, y)\right)$ is proportional to $(y-\beta)^{k}$, where $k$ depends on $\alpha$ and on a suitable factorization of $D(x)$, and $\mathbf{S r e s}_{j}$ denotes the subresultant of order $j$ (with respect to $y$ ).

This allows to symbolically construct a squarefree polynomial $g_{i}\left(\alpha_{i}, y\right)$, from every $f\left(\alpha_{i}, y\right)$, i.e., by symbolically dividing $f\left(\alpha_{i}, y\right)$ by a convenient power of $\left(y-r_{\alpha_{i}}\left(\alpha_{i}\right)\right)$, whose real roots need to be computed in the second step.

If the curve is not in generic position then we apply the linear change of coordinates

$$
x=x-y, \quad y=y,
$$

and restart the process with the new polynomial. Alternatively, one may apply a rotation with a randomly chosen angle between 0 and $\pi$. After a finite number of such transformations the curve will be in generic position. Notice that these changes of coordinates do not modify the topology of the curve, and the transformation can be reversed at the end. 
Example 1. For the bivariate polynomial

$f(x, y)=-3+12 x^{2}-18 x^{4}+12 x^{6}-3 x^{8}+12 y^{2}-28 y^{2} x^{2}+20 y^{2} x^{4}-4 y^{2} x^{6}+2 y^{4}+12 y^{4} x^{2}+2 y^{4} x^{4}-12 y^{6}+4 y^{6} x^{2}+y^{8}$,

the discriminant has real roots $\pm 1, \pm 6.236475953965871$. For $x=-1$ there are three critical points: $(-1,-2)$ and $(-1,2)$ with algebraic multiplicity 2 , and $(-1,0)$ with multiplicity 0 . Similarly for $x=1$ (see Figure 1, left). Thus, the curve is not in generic position. On the other hand, for $\alpha= \pm 6.236475953965871$, the $\mathrm{y}$-coordinate of the only double point is the complex number $\beta=8.688945478749457 i$.

If one transforms the polynomial using (22) then the leading coefficient with respect to $y$ annihilates at $x=0$, and the first condition in the generic position definition is not satisfied (see figure 1, center). By applying the transformation (22) once more, we obtain the curve in generic position (see figure 1, right).
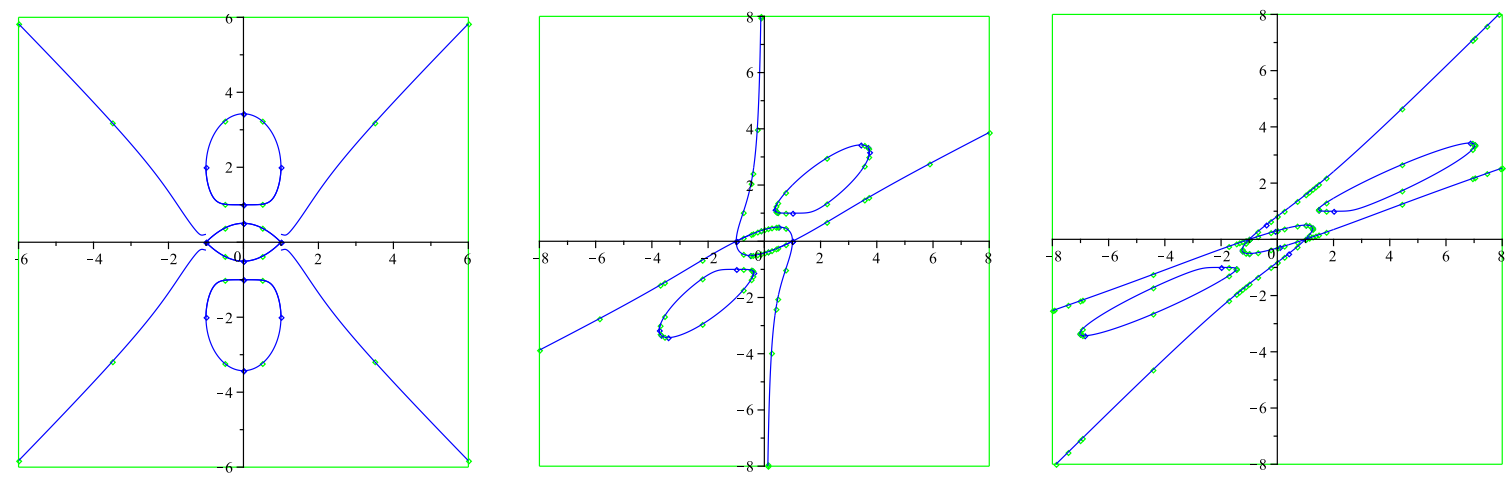

Figure 1: The curve of example 1 (left), after applying transformation (22) once (center), and twice (right).

The third step is accomplished by computing the number of real roots of the squarefree polynomials $f\left(\gamma_{i}, y\right)$ $(i \in\{1,2, \ldots, r+1\})$ with $\gamma_{1}=-\infty, \gamma_{r+1}=\infty$, and for $2 \leq i \leq r, \gamma_{i}$ being any real number in the open interval $\left(\alpha_{i-1}, \alpha_{i}\right)$. As a consequence of the generic position condition, the edges of the topological graph can be obtained using a straightforward combinatorial reasoning (see Gonzalez-Vega and Necula (2002)).

\subsection{Avoiding the discriminant computation}

Let $f(x, y)$ be a squarefree polynomial in $\mathbb{R}[x, y]$ and $\mathcal{C}_{f}$ the curve defined by the equation $f(x, y)=0$. Moreover, let $\mathbf{B}(x)=\operatorname{Bez}\left(f(x, y), f_{y}(x, y)\right)$, the Bézout matrix of $f(x, y)$ and $f_{y}(x, y)$ with respect to $y$. Note that the roots of the discriminant are the roots of $\operatorname{det}(\mathbf{B}(x))$. Let $\mathbf{C}_{0}$ and $\mathbf{C}_{1}$ be the companion matrix pencil for $\mathbf{B}(x)$. Since $f(x, y)$ is squarefree, $\operatorname{det}(\mathbf{B}(x))=\operatorname{det}\left(x \mathbf{C}_{1}-\mathbf{C}_{0}\right)$ is not identically zero.

If $n=\operatorname{deg}_{y}(f)$ and $m=\operatorname{deg}_{x}(\mathbf{B}(x))$ then $\mathbf{C}_{0}$ and $\mathbf{C}_{1}$ are square matrices of size $n m$. According to Section 2 the number of finite eigenvalues (counted with multiplicity) for the pencil $\left(\mathbf{C}_{0}, \mathbf{C}_{1}\right)$ is equal to $\operatorname{deg}(\operatorname{det}(\mathbf{B}(x)))$ while the number of infinite eigenvalues agrees with $n m-\operatorname{deg}(\operatorname{det}(\mathbf{B}(x)))$.

Assuming, as before, that $\mathcal{C}_{f}$ is in generic position, for every real eigenvalue $\alpha$ of the pencil $\left(\mathbf{C}_{0}, \mathbf{C}_{1}\right)$, in the second step of the procedure for determining the topological graph of $\mathcal{C}_{f}$, we need to compute the unique (real) multiple root of $f(\alpha, y)$ together with its multiplicity.

If $\operatorname{dim}(\operatorname{nullspace}(\mathbf{B}(\alpha)))=1$ then the normalization (with respect to the first coordinate) of any nonzero vector $v$ in nullspace $(\mathbf{B}(\alpha))$ provides $\beta$ as the second coordinate of the normalization of $v$. In this case the multiplicity of $\beta$ as a real root of $f(\alpha, y)$ is 2 (see Corollary 1 in Section 1 ).

If $\operatorname{dim}(\operatorname{nullspace}(\mathbf{B}(\alpha)))=k>1$ then, using the particular structure of the nullspace of the Bézout matrix described in (5), $\beta$ can be recovered from any basis $\mathbf{A}$ of the nullspace $(\mathbf{B}(\alpha))$, as shown by the following proposition (see Necula (2005) for a proof).

Proposition 6. If $\mathbf{A}_{i, j}$ is the $i$-th element of the $j$-th column vector of the nullspace basis of $\mathbf{B}(\alpha)$,

$$
Y_{i}=\left\{\begin{array}{cl}
0 & \text { if } \quad 1 \leq i \leq k-1 \\
(k-1) ! & \text { if } i=k
\end{array}\right.
$$


and $X$ is the solution of $\mathbf{A} X=Y$ then

$$
k ! \cdot \beta=\sum_{j=1}^{k} \mathbf{A}_{k+1, j} X_{j}
$$

In this case the multiplicity of $\beta$ as a real root of $f(\alpha, y)$ is $k+1$. In order to compute the simple roots of every $f(\alpha, y)$ and for the final determination of the topology of $\mathcal{C}_{f}$ we proceed in the same manner as described in Subsection 4.1 .

\section{Computing the topology of $f(x, y)=0$ when $f$ is presented by values}

Let $f(x, y)$ be a polynomial in $\mathbb{R}[x, y]$ whose monomial description is not available or it is costly to be either computed or used. We assume that it is possible to evaluate $f(x, y)$, and that it is known that the degrees of $f(x, y)$ with respect to $x$ and $y$ are bounded by $m$ and $n$, respectively. The curve $f(x, y)=0$ is assumed to be in generic position (see Section 4.1); later in Section 5.5 we present a method for checking whether or not a given curve is in generic position, in this context. As usual, we will also demonstrate that by making a simple linear change of coordinates we put the curve in generic position, if not already in such a position.

\subsection{Computing the $x$-coordinates of the critical points of $f(x, y)=0$}

We recall that the Bézout matrix of a pair of bivariate polynomials is a matrix polynomial. Therefore, each entry of such Bézout matrix is a univariate polynomial. The following proposition presents a degree bound for the polynomial entries of the Bézout matrix of $f(x, y)$ and $f_{y}(x, y)$ (with respect to $y$ ).

Proposition 7. Let $\boldsymbol{\sigma}=\left(\sigma_{1}<\sigma_{2}<\ldots<\sigma_{n+1}\right)$ be $n+1$ real numbers, and let $\mathbf{L}(y ; \boldsymbol{\sigma})=\left[L_{i}(y ; \boldsymbol{\sigma}): 1 \leq i \leq\right.$ $n+1]$ be the Lagrange basis of $\left[\mathbb{P}_{n}\right]^{n+1}[y]$ w.r.t. to the interpolation nodes $\boldsymbol{\sigma}$. Let $\mathbf{B}(x) \in \mathcal{M}_{n}(\mathbb{R})$ be the Bézout matrix of $f(x, y)$ and $f_{y}(x, y)$ (with respect to $y$ ) in the Lagrange basis $\mathbf{L}(y ; \tilde{\boldsymbol{\sigma}})$

$$
\mathbf{B}(x)=\mathbf{B}_{d} x^{d}+\ldots+\mathbf{B}_{0}
$$

where $B_{i} \in \mathcal{M}_{n}(\mathbb{R})$. The degree $d$ of $\mathbf{B}(x)$ is upper bounded by $2 m$

Proof. Notice that both $f(x, y)$ and $f_{y}(x, y)$ have degree at most $m$ w.r.t. $x$. From Lemma 1 , we have that $\mathbf{B}(x)=\mathbf{V B}^{M}(x) \mathbf{V}^{t}$ (where $\mathbf{B}^{M}(x)$ is the Bézout matrix of $f(x, y)$ and $f_{y}(x, y)$ w.r.t. the monomial basis, and $\mathbf{V}$ is a constant Vandermonde matrix depending on $\boldsymbol{\sigma})$. The coefficients of $\mathbf{B}^{M}(x)$ are

$$
B_{i j}^{M}=\sum_{k=1}^{\min \{i, n+1-j\}} f(x, y)_{j+k+1} f_{y}(x, y)_{i-k}-f(x, y)_{i-k} f_{y}(x, y)_{j+k+1} \text {, }
$$

then it is straightforward to conclude that the degree $\mathbf{B}(x)$ w.r.t. $x$ is bounded by $2 m$.

Proposition 8. Let $\boldsymbol{\sigma}$ and $\mathbf{L}(y ; \boldsymbol{\sigma})$ be as described in Proposition 7 . Moreover, let $\boldsymbol{\tau}=\left(\tau_{1}<\tau_{2}<\ldots<\tau_{2 m+1}\right)$ be another collection of real numbers, and $\mathbf{L}(x ; \boldsymbol{\tau})=\left[L_{i}(x ; \boldsymbol{\tau}): 1 \leq i \leq 2 m+1\right]$ be the Lagrange basis of $\mathbb{R}_{2 m}[x]$ w.r.t. the interpolation nodes $\boldsymbol{\tau}$ (the two collections of real numbers $\boldsymbol{\tau}$ and $\boldsymbol{\sigma}$ might share common nodes). Then, we have

$$
\mathbf{B}(x)=\mathbf{B}\left(\tau_{2 m+1}\right) L_{2 m+1}(x ; \boldsymbol{\tau})+\ldots+\mathbf{B}\left(\tau_{1}\right) L_{1}(x ; \boldsymbol{\tau}) .
$$

where

$$
\mathbf{B}\left(\tau_{k}\right)=\operatorname{Bez}\left(f\left(\tau_{k}, y\right), f_{y}\left(\tau_{k}, y\right)\right)=\left(\begin{array}{ccc}
b_{1,1}^{k} & \ldots & b_{1, n}^{k} \\
\vdots & & \vdots \\
b_{n, 1}^{k} & \ldots & b_{n, n}^{k}
\end{array}\right)
$$

is the $n \times n$ Bézout matrix in the Lagrange basis $\mathbf{L}(y ; \tilde{\boldsymbol{\sigma}})$ and

$$
\begin{aligned}
b_{i, j}^{k}=\frac{f\left(\tau_{k}, \sigma_{i}\right) f_{y}\left(\tau_{k}, \sigma_{j}\right)-f\left(\tau_{k}, \sigma_{j}\right) f_{y}\left(\tau_{k}, \sigma_{i}\right)}{\sigma_{i}-\sigma_{j}}, & \text { if } i \neq j, \\
b_{i, i}^{k}=f_{y}\left(\tau_{k}, \sigma_{i}\right)^{2}-f\left(\tau_{k}, \sigma_{i}\right) f_{y y}\left(\tau_{k}, \sigma_{i}\right) . & \text { otherwise. }
\end{aligned}
$$


Proof. It follows directly from Proposition 4.

To compute the values of the second derivatives $f_{y y}\left(\tau_{k}, \sigma_{i}\right)$ we use the derivative formula in Equation (16) twice by replacing the required data which are either given or have been already evaluated. Once we know the evaluations of the Bézout matrix of $f(x, y)$ and $f_{y}(x, y)$ (in the Lagrange basis $\mathbf{L}(y ; \tilde{\boldsymbol{\sigma}})$ ) at the nodes $\boldsymbol{\tau}$, the $x$-coordinates of the critical points $\left\{\alpha_{1}, \ldots, \alpha_{r}\right\}$ of the curve $f(x, y)=0$ are determined by computing the real generalized eigenvalues of the matrix pencil provided by $\mathbf{B}(x)$ as presented in Equation (23). This requires the construction of the two square matrices $\mathbf{C}_{0}, \mathbf{C}_{1} \in \mathcal{M}_{n(2 m+2)}(\mathbb{R})$. For each critical point, we shall call the vertical line $\{x=\alpha\}$ passing through it, a critical line.

\subsection{Computing the $y$-coordinate of the only critical point of $f(x, y)=0$ whose $x$-coordinate is $\alpha$}

We assume that $\alpha$ is a real generalized eigenvalue of the pencil of companion matrices $\mathbf{C}_{0}, \mathbf{C}_{1}$, such that $\mathbf{B}(\alpha)$, the corresponding Bézout matrix in the Lagrange basis is a singular matrix. If $\beta_{\alpha}$ is the unique multiple root of $f(\alpha, y)$ and $\operatorname{dim}\left(\right.$ nullspace $(\mathbf{B}(\alpha))=1$, then the nonzero column-vector $\left[L_{1}\left(\beta_{\alpha} ; \tilde{\boldsymbol{\sigma}}\right), \ldots, L_{n}\left(\beta_{\alpha} ; \tilde{\boldsymbol{\sigma}}\right)\right]^{T}$ generates the subspace nullspace $(\mathbf{B}(\alpha))$. Thus, if $\left[a_{1}, \ldots, a_{n}\right]$ is a basis of nullspace $(\mathbf{B}(\alpha))$, then from Theorem 1 we have that

$$
\left[L_{1}\left(\beta_{\alpha} ; \tilde{\boldsymbol{\sigma}}\right), \ldots, L_{n}\left(\beta_{\alpha} ; \tilde{\boldsymbol{\sigma}}\right)\right]=\frac{\left[a_{1}, \ldots, a_{n}\right]}{a_{1}+\ldots+a_{n}}
$$

and

$$
\beta_{\alpha}=\sigma_{1} L_{1}\left(\beta_{\alpha} ; \tilde{\boldsymbol{\sigma}}\right)+\ldots+\sigma_{n} L_{n}\left(\beta_{\alpha} ; \tilde{\boldsymbol{\sigma}}\right) .
$$

In this case the multiplicity of $\beta_{\alpha}$ as a real root of $f(\alpha, y)$ is 2 .

If $\operatorname{dim}(\operatorname{nullspace}(\mathbf{B}(\alpha)))=k>1$, let

$$
\mathbf{N}^{*}=\left(\begin{array}{cccc}
L_{1}\left(\beta_{\alpha} ; \tilde{\boldsymbol{\sigma}}\right) & L_{1}^{(1)}\left(\beta_{\alpha} ; \tilde{\boldsymbol{\sigma}}\right) & \ldots & L_{1}^{(k-1)}\left(\beta_{\alpha} ; \tilde{\boldsymbol{\sigma}}\right) \\
L_{2}\left(\beta_{\alpha} ; \tilde{\boldsymbol{\sigma}}\right) & L_{2}^{(1)}\left(\beta_{\alpha} ; \tilde{\boldsymbol{\sigma}}\right) & \ldots & L_{2}^{(k-1)}\left(\beta_{\alpha} ; \tilde{\boldsymbol{\sigma}}\right) \\
\vdots & \vdots & & \vdots \\
L_{n}\left(\beta_{\alpha} ; \tilde{\boldsymbol{\sigma}}\right) & L_{n}^{(1)}\left(\beta_{\alpha} ; \tilde{\boldsymbol{\sigma}}\right) & \ldots & L_{n}^{(k-1)}\left(\beta_{\alpha} ; \tilde{\boldsymbol{\sigma}}\right)
\end{array}\right)
$$

Then on the one hand, if $\mathbf{N} \in \mathcal{M}_{n \times k}(\mathbb{R})$ is a matrix whose columns are a basis of the nullspace $(\mathbf{B}(\alpha))$, then there exists a nonsingular matrix $\mathbf{A} \in \mathcal{M}_{k \times k}(\mathbb{R})$ such that

$$
\mathbf{N}=\mathbf{N}^{*} \mathbf{A} \text {. }
$$

On the other hand, let

$$
\mathbf{M}=\left(\begin{array}{cccc}
1 & 0 & \ldots & 0 \\
\beta_{\alpha} & 1 & \ldots & 0 \\
\beta_{\alpha}{ }^{2} & 2 \beta_{\alpha} & \ldots & 0 \\
\vdots & \vdots & & \vdots \\
\beta_{\alpha}{ }^{k} & k \beta_{\alpha}{ }^{k-1} & \ldots & k ! \beta_{\alpha}
\end{array}\right)
$$

having the same structure as in (5). We can take moments by multiplying by a truncated transpose of the Vandermonde matrix

$$
\mathbf{V}_{k+1, n}^{T}=\left(\begin{array}{cccc}
1 & 1 & \ldots & 1 \\
\sigma_{1} & \sigma_{2} & \ldots & \sigma_{n} \\
\sigma_{1}{ }^{2} & \sigma_{2}{ }^{2} & \ldots & \sigma_{n}{ }^{2} \\
\vdots & \vdots & & \vdots \\
\sigma_{1}{ }^{k} & \sigma_{2}{ }^{k} & \ldots & \sigma_{n}{ }^{k}
\end{array}\right) \in \mathcal{M}_{(k+1) \times n}(\mathbb{R})
$$

by considering that $\mathbf{V}_{k+1, n}^{T} \mathbf{N}^{*}=\mathbf{M}$. Thus Equation (26) can be rewritten as

$$
\mathbf{V}_{k+1, n} \mathbf{N}=\mathbf{V}_{k+1, n} \mathbf{N}^{*} \mathbf{A}=\mathbf{M A}
$$

If we define $\mathbf{Z}$ as

$$
\mathbf{Z}=\mathbf{V}_{k+1, n}^{T} \mathbf{N}
$$


we have

$$
\mathbf{Z}=\mathbf{M A},
$$

and we obtain the following result.

Proposition 9. Following the above notation,

$$
\left(\beta_{\alpha}{ }^{k}-\left(\begin{array}{l}
k \\
1
\end{array}\right) \beta_{\alpha}{ }^{k-1} \quad\left(\begin{array}{l}
k \\
2
\end{array}\right) \beta_{\alpha}{ }^{k-2} \ldots(-1)^{k-1} k \beta_{\alpha} \quad(-1)^{k}\right) \mathbf{Z}=0
$$

where $\left(\begin{array}{l}k \\ j\end{array}\right)$ denotes the binomial coefficient.

Proof. A left-inverse matrix of $\mathbf{M}$ is given by

$$
\mathbf{P}=\left(\begin{array}{cccccc}
1 & 0 & 0 & \cdots & 0 & 0 \\
-\beta_{\alpha} & 1 & 0 & \cdots & 0 & 0 \\
\frac{\beta_{\alpha}^{2}}{2} & -\beta_{\alpha} & \frac{1}{2} & \cdots & 0 & 0 \\
\vdots & \vdots & \vdots & & \vdots & \vdots \\
\frac{\left(-\beta_{\alpha}\right)^{k-1}}{(k-1) !} & \frac{\left(-\beta_{\alpha}\right)^{k-2}}{(k-2) !} & \frac{\left(-\beta_{\alpha}\right)^{k-3}}{(k-3) ! 2 !} & \cdots & \frac{1}{(k-1) !} & 0
\end{array}\right)
$$

and multiplying by $\mathbf{P}$ on the left of both sides of Equation (29) one gets the following expression for the entries of A:

$$
A_{j i}=\sum_{s=1}^{j} \frac{\left(-\beta_{\alpha}\right)^{j-s}}{(j-s) !(s-1) !} Z_{s i} .
$$

The equations in $\mathbf{M A}=\mathbf{Z}$ corresponding to the last row of $\mathbf{Z}$ have the form

$$
Z_{(k+1) i}=\beta_{\alpha}{ }^{k} A_{1 i}+k \beta_{\alpha}{ }^{k-1} A_{2 i}+k(k-1) \beta_{\alpha}{ }^{k-2} A_{3 i}+\cdots+k ! \beta_{\alpha} A_{k i} .
$$

Then, by using (31), one obtains the system (30).

The system (30) is composed by $k$ equations of degree $k$ in $\beta_{\alpha}$. By applying the Gauss method, we compute the lower triangulation $\hat{\mathbf{Z}}$ of $\mathbf{Z}$, whence

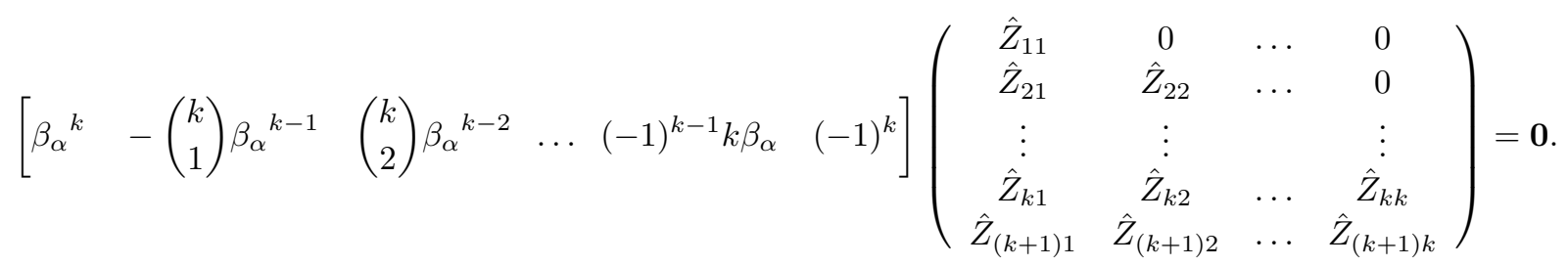

Thus, since $\hat{\mathbf{Z}}$ has rank $k$, and $\hat{Z}_{k k} \neq 0$,

$$
\beta_{\alpha}=\frac{\hat{Z}_{(k+1) k}}{k \hat{Z}_{k k}}
$$

In this case the multiplicity of $\beta_{\alpha}$ as a real root of $f(\alpha, y)$ is $k+1$.

\subsubsection{Certification of critical points}

For each eigenvalue $\alpha$ we verify if $\left|f\left(\alpha, \beta_{\alpha}\right)\right|<\varepsilon$, where $\varepsilon$ is a small tolerance value which we have chosen at the beginning; typically $\varepsilon$ is between $10^{-8}$ and $10^{-6}$. The failure of this condition may be due to two reasons: numerical error, or a complex, and non-real root of the discriminant. The first problem is solved by increasing the number of precision digits, and repeating the computation of the generalized eigenvalues. To analyze the second case, when the discriminant has complex and non-real roots, we consider the following example; for

$$
f(x, y)=\left(3 x^{2}+4 y+4 y^{2}\right)\left(x^{2}-2 y+y^{2}\right)\left(x^{2}+2 x+2 y^{2}\right)\left(x^{2}-2 x+y^{2}\right),
$$


$f(6, y)$ and $f_{y}(6, y)$ have two common complex roots which are conjugate. Hence $x=6$ is one of the computed eigenvalues, but the curve does not satisfy the second condition in the Definition 7 of generic position. Thus, similarly to Section 4.1, we make a linear change of coordinates (a rotation or the transformation (22)) and restart the algorithm with the new polynomial. If we use the transformation (22) to obtain the values of the new polynomial $\hat{f}(x, y)=f(x-y, y)$ at the nodes $\boldsymbol{\tau}, \boldsymbol{\sigma}$ we need to apply the following proposition.

Proposition 10. Let

$$
p(x)=\sum_{i=1}^{d+1} p\left(\tau_{i}\right) L_{i}(x ; \boldsymbol{\tau})
$$

be a polynomial expressed in the Lagrange basis. If $\hat{p}(x)=p(x-a)$, then

$$
\hat{p}\left(\tau_{i}\right)=\sum_{j=1}^{d+1} p\left(\tau_{j}\right) L_{j}\left(\tau_{i}-a ; \boldsymbol{\tau}\right) .
$$

Proof. The expression of $\hat{p}$ in the Lagrange basis $\mathbf{L}(x ; \boldsymbol{\tau})$ is

$$
\hat{p}(x)=\sum_{i=1}^{d+1} \hat{p}\left(\tau_{i}\right) L_{i}(x ; \boldsymbol{\tau}) .
$$

On the other hand,

$$
p(x-a)=\sum_{j=1}^{d+1} p\left(\tau_{j}\right) L_{j}(x-a ; \boldsymbol{\tau})=\sum_{j=1}^{d+1} p\left(\tau_{j}\right) \sum_{i=1}^{d+1} L_{j}\left(\tau_{i}-a ; \boldsymbol{\tau}\right) L_{i}(x ; \boldsymbol{\tau})=\sum_{i=1}^{d+1}\left[\sum_{j=1}^{d+1} p\left(\tau_{j}\right) L_{j}\left(\tau_{i}-a ; \boldsymbol{\tau}\right)\right] L_{i}(x ; \boldsymbol{\tau})
$$

and the proposition follows.

As a consequence

$$
\hat{f}\left(\tau_{k}, \sigma_{j}\right)=\sum_{i=1}^{2 m+1} f\left(\tau_{i}, \sigma_{j}\right) L_{i}\left(\tau_{k}-\sigma_{j} ; \boldsymbol{\tau}\right)
$$

and

$$
\hat{f}\left(x, \sigma_{j}\right)=\sum_{k=1}^{2 m+1}\left[\sum_{i=1}^{2 m+1} f\left(\tau_{i}, \sigma_{j}\right) L_{i}\left(\tau_{k}-\sigma_{j} ; \boldsymbol{\tau}\right)\right] L_{k}(x ; \boldsymbol{\tau})
$$

\subsection{Computing the simple solutions of $f(\alpha, y)=0$}

We assume that, for fixed $\alpha$, we know the value of $\beta_{\alpha}$ such that $\left(\alpha, \beta_{\alpha}\right)$ is a critical point of $f(x, y)$, and also the multiplicity $k+1$ of $\beta_{\alpha}$ as a root of $f(\alpha, y)$. We can proceed to get the rest of the roots of the polynomial $f(\alpha, y)$. Remember that these roots are simple because $f(x, y)$ is in generic position.

Notice that we can easily obtain the Lagrange presentation of the polynomial

$$
F^{\alpha}(y)=\frac{f(\alpha, y)}{\left(y-\beta_{\alpha}\right)^{k+1}}
$$

w.r.t. the Lagrange basis $\mathbf{L}(y ; \tilde{\boldsymbol{\sigma}})$ where $\tilde{\boldsymbol{\sigma}}$ is a subset of $n-k$ elements of $\boldsymbol{\sigma}$, avoiding to choose any $\sigma_{i}$ that is equal or too close to $\beta_{\alpha}$. The coefficients of the polynomial in the Lagrange basis are

$$
\left\{\frac{f\left(\alpha, \sigma_{i}\right)}{\left(\sigma_{i}-\beta_{\alpha}\right)^{k+1}}: \sigma_{i} \in \tilde{\boldsymbol{\sigma}}\right\} .
$$

With these coefficients we construct the corresponding pencil of companion matrices, which in this case are constant matrices of size $n-k+2$, and find the generalized eigenvalues. Then we eliminate non-real complex and infinite eigenvalues. The remaining eigenvalues are the real simple solutions of $f(\alpha, y)=0$. 


\subsection{Determining the edges of the graph}

The points on all the critical lines obtained by using the method described in the previous sections will be the vertices of the topological graph. To determine which pairs of vertices in two consecutive critical lines must be joined by edges, we first choose a sequence $\gamma_{1}, \ldots, \gamma_{r+1}$ of values of $x$ such that

$$
\gamma_{1}<\alpha_{1}, \quad \alpha_{i-1}<\gamma_{i}<\alpha_{i}, i=2, \ldots, r, \quad \alpha_{r}<\gamma_{r+1} .
$$

Then, we compute the solutions of $f\left(\gamma_{i}, y\right)=0$ for each $i$. Let $b_{i}$ denote the number of such solutions and $\nu_{i}$ be the number of simple points in the critical line $\left\{x=\alpha_{i}\right\}$. Then, the number of edges going from the critical point $\left(\alpha_{i}, \beta_{\alpha_{i}}\right)$ to the left is equal to $b_{i}-\nu_{i}$, and the number of edges to the right is $b_{i+1}-\nu_{i}$. From each of the other points in the critical line there is only one edge to the left and only one to the right because they are simple points. Figure 2 shows an example with $b_{i}=7, \nu_{i}=3, b_{i+1}=5$.

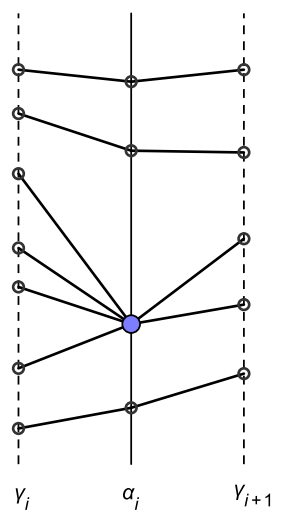

Figure 2: Example of edges: $b_{i}=7, \nu_{i}=3, b_{i+1}=5$

The choice of the intermediate values $\gamma_{i}, i=2, \ldots, r$, is made based on the following criterium: if there is a node $\tau_{j}$ in the interval $\left(\alpha_{i-1}, \alpha_{i}\right)$, we choose it, otherwise, we choose the midpoint of the interval. Similarly, $\gamma_{1}$ is either a node or equal to $\alpha_{1}-1$, and also $\gamma_{r+1}$ is either a node or equal to $\alpha_{r}+1$.

To compute the real roots of $f\left(\gamma_{i}, y\right)$ we proceed analogously to Section 5.3 ; evaluate $f\left(\gamma_{i}, \sigma_{j}\right), j=1, \ldots, n+1$, build the companion matrix pencil, and solve for the generalized eigenvalues.

\subsection{Checking generic position}

In some cases, the nongeneric position of the curve is detected when certifying the critical points (see 5.2.1). In other cases, one has to check conditions 1 and 2 in Definition 7. To check the first condition, for each generalized eigenvalue $\alpha$ we must determine that the leading coefficient of $f(x, y)$ with respect to $y$ does not vanish when $x=\alpha$. To obtain the leading coefficient we use the following proposition whose proof follows directly from Definition 4.

Proposition 11. Let $p(y)$ be a polynomial of degree $d$ and $\boldsymbol{\sigma}=\left(\sigma_{1}, \ldots, \sigma_{d+1}\right)$ be a collection of real numbers. The leading coefficient of $p(y)$ is

$$
a_{d}=\sum_{i=1}^{d+1} \omega_{i}(\boldsymbol{\sigma}) p_{i},
$$

where $\omega_{i}(\boldsymbol{\sigma})$ is given by Formula (8), and $p_{i}=p\left(\sigma_{i}\right)$.

Therefore, in our case, for each generalized eigenvalue $\alpha$ we compute

$$
L C_{\alpha}=\sum_{i=1}^{n+1} \omega_{i}(\boldsymbol{\sigma}) \sum_{j=1}^{2 m+1} f\left(\tau_{j}, \sigma_{i}\right) L_{j}(\alpha, \boldsymbol{\tau}) .
$$


If for some $\alpha$ we have that $\left|L C_{\alpha}\right|<\varepsilon$ then the curve is not in generic position (it has a vertical asymptote at $x=\alpha)$.

The method to verify the second condition in the generic position definition is based on Proposition 2 and Corollary 1. First, it is necessary to find the $x$-coordinates of the critical points $\left\{\alpha_{1}, \ldots, \alpha_{r}\right\}$ as in Section 5.1 . For each $\alpha_{j}$ we compute the nested finite sequence of linear spaces

$$
N_{k}=\bigcap_{s=1}^{k} \text { nullspace }\left(\operatorname{Bez}_{y}\left(f\left(\alpha_{j}, y\right), \frac{\partial^{s} f}{\partial y^{s}}\left(\alpha_{j}, y\right)\right)\right)
$$

ending when $\operatorname{dim}\left(N_{i}\right) \leq 1$.

Notice that $\frac{\partial^{i} f}{\partial y^{i}}\left(\alpha_{j}, y\right)$ can be computed using the nodes $\boldsymbol{\sigma}$ and applying the formula (16) $i$ times. The corresponding Bézout matrix is given by Proposition 4.

As soon as $\operatorname{dim}\left(N_{s+1}\right)-\operatorname{dim}\left(N_{s}\right)>1$ for one $\alpha_{j}$, the computation is stopped and we conclude that the curve is not in generic position. In this case, we perform a linear transformation on $f(x, y)$ as explained in 4.1 and 5.2 .1 , and restart the algorithm by using the values of the transformed polynomial.

\subsection{The algorithm}

\section{Input:}

- The degree bounds $m$ and $n$ for $f(x, y)$ with respect to $x$ and $y$, respectively.

- The nodes for $x, \boldsymbol{\tau}=\left(\tau_{1}<\ldots<\tau_{2 m+1}\right)$, for $y, \boldsymbol{\sigma}=\left(\sigma_{1}<\ldots<\sigma_{n+1}\right)$, and the corresponding values $\left(f\left(\tau_{i}, \sigma_{j}\right): 1 \leq i \leq 2 m+1,1 \leq j \leq n+1\right)$.

- The tolerance $\varepsilon$.

Output: The graph representing the topology of $f(x, y)=0$.

1. Critical points

(a) For $k=1, \ldots, 2 m+1$ compute the matrices $\mathbf{B}_{k}$ by using Formula (24).

(b) Construct the companion matrix pencil $\left(\mathbf{C}_{0}, \mathbf{C}_{1}\right)$ and compute its generalized eigenvalues. Discard all complex non-real and infinite eigenvalues. The remaining eigenvalues are $\left\{\alpha_{1}<\ldots<\alpha_{r}\right\}$.

(c) Detection of vertical asymptotes: For each eigenvalue $\alpha_{i}$, compute $L C_{\alpha_{i}}$ as in equation (34). If for any $\alpha_{i},\left|L C_{\alpha_{i}}\right|<\varepsilon$, then a linear transformation must be applied to the curve (see Section 5.5), and the algorithm must be restarted with the transformed curve.

(d) For each eigenvalue $\alpha_{i}$, compute the corresponding $y$-coordinate $\beta_{i}$ as in Section 5.2. Check if $\left|f\left(\alpha_{i}, \beta_{i}\right)\right|<\varepsilon$; if this condition is not satisfied for one $\alpha_{i}$ then

- Increase the computing precision by 10 digits and repeat the computation of the critical points.

- If the precision has been increased sufficiently (up to 200 digits) but the desired condition is not yet satisfied conclude that the curve is not in generic position. In this case, apply a linear transformation as explained in Section 5.5 and restart the algorithm with the transformed polynomial.

\section{Checking generic position}

For each eigenvalue $\alpha_{i}$ find a basis of each subspace $N_{k}$ as defined in (35) by using the Singular Value Decomposition on the matrix in Equation (6). If for some $s, \operatorname{dim}\left(N_{s}\right)-\operatorname{dim}\left(N_{s-1}\right)>1$, then the curve is not in generic position, a linear transformation must be applied to the curve (see Section 5.5), and the algorithm must be restarted with the transformed curve.

3. Other points on a critical line

For each eigenvalue $\alpha_{i}$, let $k_{i}+1$ be the multiplicity of $\beta_{i}$ as a root of $f\left(\alpha_{i}, y\right)$. Then

(a) Construct the matrix pencil associated with the nodes $\left(\sigma_{j}: 1 \leq j \leq n-k_{i}\right)$ and the values

$$
\left(\frac{f\left(\alpha_{i}, \sigma_{j}\right)}{\left(\sigma_{j}-\beta_{i}\right)^{k_{i}+1}}: 1 \leq j \leq n-k_{i}\right) .
$$

Compute the generalized eigenvalues and discard those complex non-real and infinite. 
(b) For each eigenvalue $y *$, the point $\left(\alpha_{i}, y *\right)$ is one of the regular points of the curve on the considered critical line.

(c) Let $\nu_{i}$ be the number of simple points on each critical line.

\section{Points on the noncritical lines}

(a) Choose intermediate $x$-coordinates $\gamma_{1}<\alpha_{1}, \alpha_{i-1}<\gamma_{i}<\alpha_{i}, i=2, \ldots, r, \alpha_{r}<\gamma_{r+1}$, in the following way:

i. If there is a node $\tau_{j}<\alpha_{1}-0.3$ let $\gamma_{1}$ be the biggest of such nodes; otherwise, let $\gamma_{1}=\alpha_{1}-1$.

ii. For $i=2, \ldots, r$, if there is a node $\tau_{j} \in\left(\alpha_{i-1}+\left(\alpha_{i}-\alpha_{i-1}\right) / 4, \alpha_{i-1}+3\left(\alpha_{i}-\alpha_{i-1}\right) / 4\right)$ let $\gamma_{i}$ be any such node; otherwise let $\gamma_{i}=\left(\alpha_{i-1}+\alpha_{i}\right) / 2$.

iii. If there is a node $\tau_{j}>\alpha_{r}+0.3$ let $\gamma_{1}$ be the smallest of such nodes; otherwise, let $\gamma_{r}=\alpha_{r}+1$. The constant 0.3 above is to avoid using a node too close to $\alpha_{1}$ or $\alpha_{r}$; it has been chosen heuristically, for better visualization of the graph, and to avoid numerical problems.

(b) For each $\gamma_{i}$, construct the companion matrix pencil associated with the nodes $\left(\sigma_{j}: 1 \leq j \leq n+1\right)$ and the values $\left(f\left(\gamma_{i}, \sigma_{j}\right): 1 \leq j \leq n+1\right)$. Compute the generalized eigenvalues and discard those complex non-real and infinite.

(c) Let $b_{i}$ be the number of these simple points with $x$-coordinate equal to $\gamma_{i}$.

\section{Edges of the graph}

Starting from the lowest point, connect each simple point to the next available point on the line $x=\gamma_{i}$ and to the next available point on the line $x=\gamma_{i+1}$ until the critical point is reached (see figure 2). Connect the critical point to $b_{i}-\nu_{i}$ points on the line $x=\gamma_{i}$ and to $b_{i+1}-\nu_{i}$ points on the line $x=\gamma_{i+1}$. Then, continue connecting the remaining simple points to one point on the left and one point on the right.

Example 2. To illustrate the main steps of the algorithm we use the algebraic curve obtained by applying the transformation in Equation (22) to the curve in Example 1 (plotted in Figure 1, right).

Input:

$m=8, \quad n=8, \quad \boldsymbol{\tau}=(-8,-7,-6,-5,-4,-3,-2,-1,0,1,2,3,4,5,6,7,8), \quad \boldsymbol{\sigma}=(-4,-3,-2,-1,0,1,2,3,4)$,

The corresponding values are in the following matrix, where the $i j$-element is $f\left(\tau_{i}, \sigma_{j}\right)$ :

$\left.\begin{array}{llllllllll}17085 & 15552 & -183795 & -4660416 & -47258883 & -291960000 & -1299890163 & -4594916160 & -13699084611 \\ 36864 & 2025 & -12288 & -1044375 & -15925248 & -124862391 & -648744960 & -2553167223 & -8235479040 \\ 102093 & -1920 & 1437 & -162816 & -4501875 & -48218112 & -303025635 & -1354366848 & -4783887411 \\ 196608 & 2025 & 0 & -13527 & -995328 & -16343607 & -130621440 & -680410935 & -2671706112 \\ 120189 & 15552 & -435 & -192 & -151875 & -4660416 & -50973363 & -320397120 & -1425581187 \\ -983040 & 19977 & 0 & 9 & -12288 & -1044375 & -17522688 & -139475415 & -720961536 \\ -5708211 & -155520 & 1437 & 0 & -243 & -162816 & -5091555 & -55064448 & -341940915 \\ -20508672 & -1255095 & -12288 & 9 & 0 & -13527 & -1167360 & -19163223 & -149876736 \\ -59394627 & -5609280 & -183795 & -192 & -3 & -192 & -183795 & -5609280 & -59394627 \\ -149876736 & -19163223 & -1167360 & -13527 & 0 & 9 & -12288 & -1255095 & -20508672 \\ -341940915 & -55064448 & -5091555 & -162816 & -243 & 0 & 1437 & -155520 & -5708211 \\ -720961536 & -139475415 & -17522688 & -1044375 & -12288 & 9 & 0 & 19977 & -983040 \\ -1425581187 & -320397120 & -50973363 & -4660416 & -151875 & -192 & -435 & 15552 & 120189 \\ -2671706112 & -680410935 & -130621440 & -16343607 & -995328 & -13527 & 0 & 2025 & 196608 \\ -4783887411 & -1354366848 & -303025635 & -48218112 & -4501875 & -162816 & 1437 & -1920 & 102093 \\ -8235479040 & -2553167223 & -648744960 & -124862391 & -15925248 & -1044375 & -12288 & 2025 & 36864 \\ -13699084611 & -4594916160 & -1299890163 & -291960000 & -47258883 & -4660416 & -183795 & 15552 & 17085\end{array}\right)$


Critical points.

The companion matrix pencil matrices are of size $144 \times 144$, and the real and finite generalized eigenvalues (without counting multiplicities) are

$$
\{ \pm 7.0217988481097227684, \pm 1.4608761201425284718, \pm 1.2531054640051353026, \pm 1\} .
$$

The corresponding critical points are

$$
\begin{aligned}
& ( \pm 7.0217988481097227684, \pm 3.3476041758252916187), \\
& ( \pm 1.4608761201425284718, \pm 1.0577307224691838865), \\
& ( \pm 1.2531054640051353026, \pm 0.38099545231238395034),
\end{aligned}
$$

with multiplicity 2 and $( \pm 1,0)$ with multiplicity 4.

Other points on critical lines.

For each of the eight critical points, we construct the corresponding companion matrix pencil and compute the generalized eigenvalues. They give the following points:

On the lines $x= \pm 7.0217988481097227684$ :

$$
\begin{aligned}
& ( \pm 7.0217988481097227684, \pm 7.1617016141985190797) ; \\
& ( \pm 7.0217988481097227684, \pm 2.1915651133634752340) .
\end{aligned}
$$

On the lines $x= \pm 1.4608761201425284718$ :

$$
\begin{aligned}
& ( \pm 1.4608761201425284718, \pm 1.9547205884882777201) ; \\
& ( \pm 1.4608761201425284718, \pm 0.15719056570656257280)
\end{aligned}
$$

On the lines $x= \pm 1.2531054640051353026$ :

$$
\begin{aligned}
& ( \pm 1.2531054640051353026, \pm 1.7851493909851024819) ; \\
& ( \pm 1.2531054640051353026, \pm 0.085491388179816599602) .
\end{aligned}
$$

On the lines $x= \pm 1$ :

$$
\begin{aligned}
& ( \pm 1, \pm 1.5830673160968719153) \\
& ( \pm 1, \pm 0.50534806187044243447)
\end{aligned}
$$

Thus $\nu_{i}=2$, for $i=1, \ldots, 8$.

Noncritical lines and edges.

The nine intermediate noncritical lines used to compute the edges of the graph are the following: $x=-8$, $x=-5, x=-1.3569907920738318872, x=-1.1265527320025676513, x=0, x=1.1265527320025676513$, $x=1.3569907920738318872, x=3, x=8$. The numbers of points on each noncritical line are $b_{i}=$ $\{2,4,2,4,4,4,2,4,2\}$.

This example requires a precision of 20 digits because there are pairs of eigenvalues which are rather close. The graph is represented in Figure 3.

\subsection{Experimental results}

The following analysis can be used as an indication of the computational cost of the introduced method to compute the topology of $f(x, y)=0$ with $m$ and $n$ denoting the degrees of $f(x, y)$ with respect to $x$ and $y$, respectively. Recall that the number of critical points is $r \leq 2 \mathrm{~m}$. In the algorithm, the number of evaluations of $f(x, y)$, using the Lagrange basis, is

$$
\text { number of evaluations }=(r+1) n+r \leq(2 m+1) n+2 m=2 m(n+1)+n .
$$

The number of generalized eigenvalue problems to solve is the following:

- For finding the $\alpha_{i}, 1 \leq i \leq r$, we solve one generalized eigenvalue problem for a companion matrix pencil of size $2 n(m+1)$. 


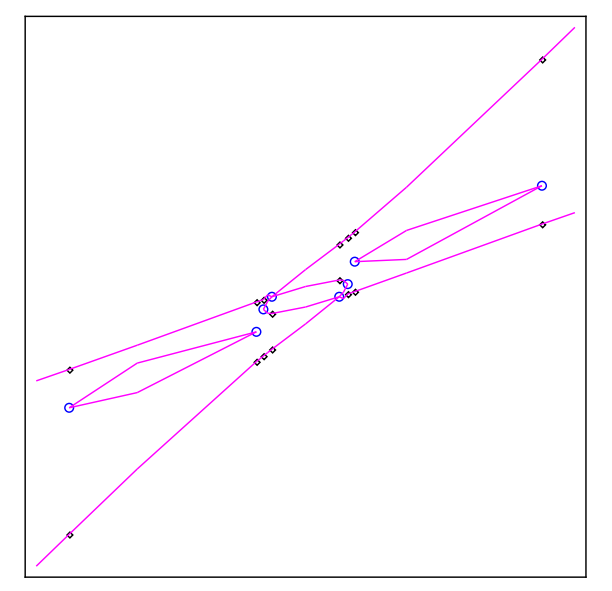

Figure 3: The graph of the algebraic curve in example 2; the circled points are critical points, and the diamonds are simple points on critical lines.

\begin{tabular}{|c||c|c|c|c|c|c|c|c|c|c|}
\hline Ex. & $m$ & $n$ & min value & $\max$ value & size of $\mathbf{C}_{i}$ & precision & time $_{1}$ & time $_{2}$ & time & Total \\
\hline \hline 1 & 6 & 6 & 0 & 383724 & 84 & 15 & 0.016 & 32.140 & 0.344 & 32.500 \\
2 & 25 & 1 & 1 & $\sim 8.8 \times 10^{34}$ & 52 & 10 & 0.062 & 4.734 & 0.016 & 4.812 \\
3 & 4 & 4 & 0 & 488 & 40 & 10 & 0.032 & 0.547 & 0.265 & 0.844 \\
4 & 6 & 7 & 0 & 11363328 & 98 & 20 & 0.031 & 138.312 & 1.016 & 139.359 \\
5 & 6 & 8 & 0 & 156381.84 & 112 & 20 & 0.032 & 237.812 & 4.860 & 242.704 \\
6 & 7 & 8 & 0 & 18416005 & 128 & 20 & 0.047 & 286.703 & 9.422 & 296.172 \\
7 & 7 & 7 & 0 & 9603789 & 112 & 15 & 0.031 & 131.859 & 0.641 & 132.531 \\
8 & 6 & 6 & 0.01 & 303271775.99 & 84 & 25 & 0.031 & 31.265 & 1.235 & 32.532 \\
\hline
\end{tabular}

Table 1: Experimental results

- For determining the solutions of each $f\left(\alpha_{i}, y\right)=0$ we solve $r$ generalized eigenvalue problems for companion matrix pencils of size $n-k_{i}+2 \leq n+1$.

- For computing the simple points in every non-critical line we solve $r+1$ generalized eigenvalue problems for companion matrix pencils of size $n+2$.

For testing purposes the algorithm has been implemented in Maple, and several examples have been analyzed. As a sample, in table 1 we summarize the computing times for eight examples. The information provided for each example contains the degrees $m=\operatorname{deg}_{x}(f)$ and $n=\operatorname{deg}_{y}(f)$, the size of the companion matrix pencil $\mathbf{C}_{0}, \mathbf{C}_{1}$, the minimum and the maximum of all $\left|f\left(\tau_{i}, \sigma_{j}\right)\right|$ (min value and max value, respectively) the number of digits used in the computations (precision) and the computing times in seconds; time ${ }_{1}$ is the time spent in the evaluations, the construction of the Bézout matrix and the construction of $\mathbf{C}_{0}, \mathbf{C}_{1}$. time $e_{2}$ is the time spent in computing the generalized eigenvalues and $t_{i m e}$ is the time spent in computing the necessary points and drawing the graph.

The corresponding examples have been chosen from Manocha and Demmel (1995), Gonzalez-Vega and Necula (2002) and Labs (2010). The graphs of these examples are presented in figures 4 and 5. To show that the curve of example 5 has three closed components, we show two zoom view of details in figure 6 . This example illustrates the accuracy of the method since it distinguish different critical points which are very close to each other. It is clear that most of the computation time is taken by the computation of the generalized eigenvalues. The companion matrix pencils are very big, but they are highly structured and sparse. For the testing we have used the Eigenvalues procedure provided by Maple, but an ad-hoc procedure that takes advantage of the sparseness of the companion matrix pencil would considerably improve efficiency.

The topology of the same examples have been computed using the Top algorithm presented in GonzalezVega and Necula (2002). The results are shown in table 2. The big difference in performance is due to the 

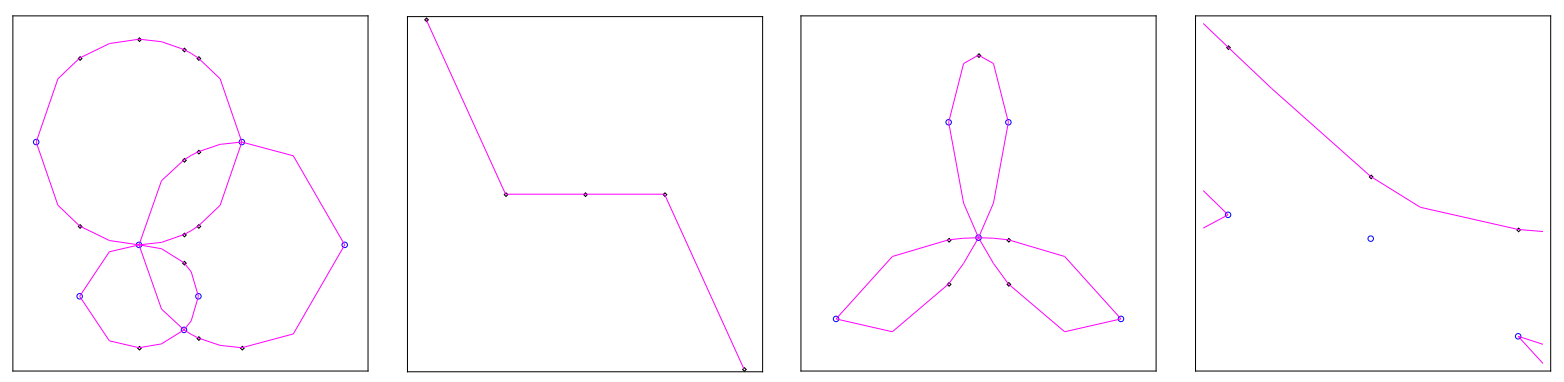

Figure 4: Examples 1, 2, 3 and 4 for $f(x, y)=0$.
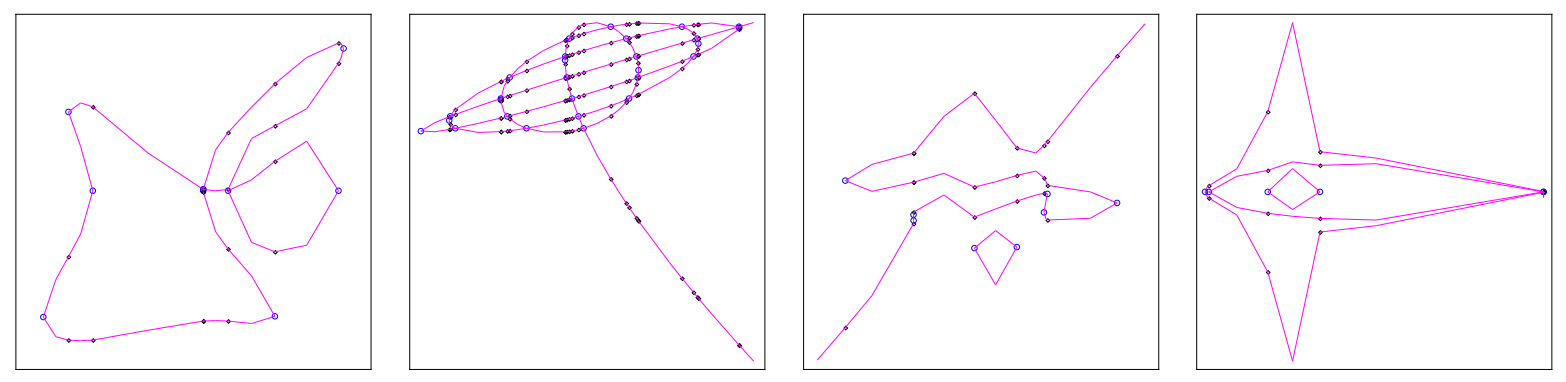

Figure 5: Examples 5, 6, 7 and 8 for $f(x, y)=0$.

computation of the generalized eigenvalues. The accuracy of the resulting topology is the same with both algorithms.

\section{Computing the topology of $(x(t), y(t))$ when $x(t)$ and $y(t)$ are presented by values}

In this section we consider the same problem as in Section 5. However, the curves to be considered in this section are neither known implicitly nor by explicit parametric descriptions but defined parametrically only by some data samples (coming from their parameterizations). Let $\mathcal{C}$ be a real curve parametrized by $\varphi(t)=(x(t), y(t)), t \in \mathbb{R}$, where $x(t)$ and $y(t)$ are polynomials described by values. In addition, we suppose that the curve is proper. Recall that a parametrization is said to be proper if it is injective for almost all the points of $\mathcal{C}$. That implies that there is at most a finite number of points of $\mathcal{C}$ generated by more than one value of the parameter $t$.

Our goal is to obtain the topology of $\mathcal{C}$ assuming that the parameterization is not available or costly to be either computed or used. Instead it is possible to compute their evaluations. Moreover, it is known that the degrees of $x(t)$ and $y(t)$ with respect to $t$ are respectively bounded by $m$ and $n$, and $d=\max \{m, n\}$.

\begin{tabular}{|c||c|c|c|c|c|c|c|}
\hline Ex. & $m$ & $n$ & time $_{1}$ & time $_{2}$ & time $_{3}$ & by values time & Top time \\
\hline \hline 1 & 6 & 6 & 0.016 & 32.140 & 0.344 & 32.500 & 0.171 \\
2 & 25 & 1 & 0.062 & 4.734 & 0.016 & 4.812 & 0.05 \\
3 & 4 & 4 & 0.032 & 0.547 & 0.265 & 0.844 & 0.078 \\
4 & 6 & 7 & 0.031 & 138.312 & 1.016 & 139.359 & 0.031 \\
5 & 6 & 8 & 0.032 & 237.812 & 4.860 & 242.704 & 0.187 \\
6 & 7 & 8 & 0.047 & 286.703 & 9.422 & 296.172 & 0.452 \\
7 & 7 & 7 & 0.031 & 131.859 & 0.641 & 132.531 & 0.125 \\
8 & 6 & 6 & 0.031 & 31.265 & 1.235 & 32.532 & 0.078 \\
\hline
\end{tabular}

Table 2: Comparison with Top algorithm. 

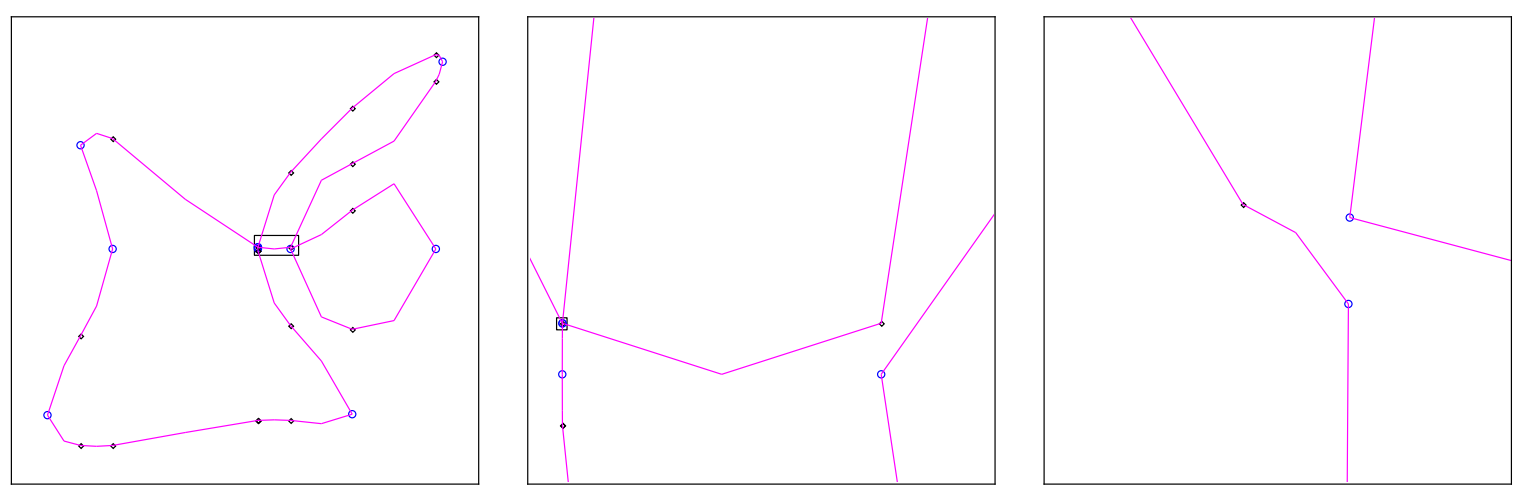

Figure 6: Zooming details of Example 5 showing the separation of components.

\subsection{Using the parametric expression}

In this section, to compute the topology of planar curves parametrized by polynomials we follow the methodology presented in Alcázar and Díaz-Toca (2010). However, unlike Alcázar and Díaz-Toca (2010), here, we assume that the parameterization is not available or costly to be either computed or used.

\subsubsection{Computation of critical points}

The set of the critical points of the curve $\mathcal{C}$ is formed by ramification points and singular points. The ramification points are those points satisfying $x^{\prime}(t)=0$ but $y^{\prime}(t) \neq 0$. The singular points include the points where $x^{\prime}(t)=y^{\prime}(t)=0$ and also the self-intersection points of the curve.

Computation of t-values which generate singular points.

Following the above notation, in Pérez-Díaz (2007) one can find the following result which characterizes the singular points of a rational parametrization. To state the result precisely we introduce some notation which are used throughout this section. Let

$$
p(t, s)=\frac{x(t)-x(s)}{t-s}, \quad q(t, s)=\frac{y(t)-y(s)}{t-s}, \quad \mathrm{r}(s)=\operatorname{resultant}_{t}(p, q) .
$$

Observe that $p(t, s)$ and $q(t, s)$ are the Cayley quotient of $x(t)$ and 1 , and $y(t)$ and 1 , respectively. Moreover $\max \left(\operatorname{deg}_{t}(p), \operatorname{deg}_{t}(q)\right)=d-1$. The resultant of $p(t, s)$ and $q(t, s)$, with respect to $t, \mathrm{r}(s)$, is called the $D$-resultant or Taylor resultant of $x(t)$ and $y(t)$ (see van den Essen and Yu (1997) and Abhyankar (1990)).

Theorem 2. Let $\varphi(t)$ be a parametrization of $\mathcal{C}$ and let $P_{0} \in \mathcal{C}$ be an affine singularity of $\mathcal{C}$ reachable by some value $s_{0} \in \mathbb{C}$ of the parameter. Then, $\mathrm{r}\left(s_{0}\right)=0$.

Since $\mathrm{r}(s)$ is proportional to the determinant of the matrix $\operatorname{Bez}_{t}(p, q)$, we can obtain the values of the parameter which generate singularities by applying the theory described in Section 3 , as follows.

Observe first that as a consequence of the special form of $p(t, s)$ and $q(t, s)$, the degree of $\mathbf{B}(s)$ is $d-2($ for details, see Diaz-Toca et al. (2012)). Then, let $\boldsymbol{\tau}=\left(\tau_{1}<\ldots<\tau_{d+1}\right)$ and $\boldsymbol{\sigma}=\left(\sigma_{1}<\ldots<\sigma_{d-1}\right)$ be two disjoint collections of real numbers. Suppose that the values $\left(x\left(\tau_{i}\right), y\left(\tau_{i}\right)\right)$ and $\left(x\left(\sigma_{i}\right), y\left(\sigma_{i}\right)\right)$ are given or easy to compute for $i=1, \ldots, d+1$. Thus, the expression of the Bézout matrix of $p(t, s)$ and $q(t, s)$ with respect to $t$ in the Lagrange basis is

$$
\mathbf{B}(s)=\operatorname{Bez}_{t}(p(t, s), q(t, s))=\sum_{k=1}^{d-1} \mathbf{B}_{k} L_{k}(s ; \boldsymbol{\sigma}),
$$

where $\mathbf{B}_{k}$ is a $(d-1) \times(d-1)$ numerical matrix. According to Proposition 4 , the entries of the matrix $\mathbf{B}_{k}$ $=\operatorname{Bez}_{t}\left(p\left(t, \sigma_{k}\right), q\left(t, \sigma_{k}\right)\right)=\left(b_{i j}\right)_{i, j=1, \ldots, d-1}$, for $1 \leq k \leq d-1$, are given by

$$
\begin{aligned}
b_{i j} & =\frac{\left[x\left(\tau_{i}\right)-x\left(\sigma_{k}\right)\right]\left[y\left(\tau_{j}\right)-y\left(\sigma_{k}\right)\right]-\left[x\left(\tau_{j}\right)-x\left(\sigma_{k}\right)\right]\left[y\left(\tau_{i}\right)-y\left(\sigma_{k}\right)\right]}{\left(\tau_{i}-\sigma_{k}\right)\left(\tau_{j}-\sigma_{k}\right)\left(\tau_{i}-\tau_{j}\right)} & \text { if } i \neq j, \\
b_{i i} & =\frac{x^{\prime}\left(\tau_{i}\right)\left(y\left(\tau_{i}\right)-y\left(\sigma_{k}\right)\right)-y^{\prime}\left(\tau_{i}\right)\left(x\left(\tau_{i}\right)-x\left(\sigma_{k}\right)\right)}{\left(\tau_{i}-\sigma_{k}\right)^{2}} & \text { otherwise. }
\end{aligned}
$$


As before, the values of $x^{\prime}\left(\tau_{i}\right)$ and $y^{\prime}\left(\tau_{i}\right), i=1, \ldots, d-1$, can be computed by using the formula (16). Therefore, to obtain the matrices $\mathbf{B}_{k}, k=1, \ldots, d-1$, we only need the values $\left(x\left(\tau_{i}\right), y\left(\tau_{i}\right)\right), i=1, \ldots, d+1$ and $\left(x\left(\sigma_{i}\right), y\left(\sigma_{i}\right)\right), i=1, \ldots, d-1$.

Next we construct the companion matrix pencil $\left(\mathbf{C}_{0}, \mathbf{C}_{1}\right)$ (see Definition 5) and determine its generalized eigenvalues. Notice that there are two possibilities:

1. There are no eigenvalues, which means that the resultant $r(s)$ is a constant polynomial. If $r(s)=0$ then the parametrization is not proper and so our methodology cannot be applied; else, i.e., $\mathrm{r}(s) \neq 0$ then, there are no singular points.

2. There are eigenvalues; in this case, we discard complex non-real and infinite eigenvalues and those remaining are the parameter values which generate the affine singularities of the curve.

Computation of t-values which generate ramification points.

To find the ramification points one must solve the following equation

$$
x^{\prime}(t)=0 .
$$

Since we do not have the polynomial expression of $x(t)$ in the monomial basis, to solve this equation we construct the corresponding companion matrix pencil $\left(\mathbf{C}_{0}, \mathbf{C}_{1}\right)$ from the values $x^{\prime}\left(\tau_{i}\right)$ at the nodes. In this case, the companion matrix pencil is of size $m+1$. Then, we compute the generalized eigenvalues and discard those complex non-real and infinite.

Computation of critical points.

The coordinates of the critical points are obtained by substituting the values of $t$ in the evaluation formula in Definition 4. We shall take into consideration that those eigenvalues which generate the singular and ramification points must be grouped together. In other words, we need to deal with the eigenvalue clustering problem (i.e., we must distinguish those eigenvalues coming from the same roots). To achieve this goal we follow the methodology introduced in Corless et al. (2002).

Once we obtain the finite real generalized eigenvalues, we then need to compute the coordinates of the corresponding points; to compute the correct number of critical points, we must, on the one hand, obtain the $x$-coordinates of the critical points, and on the other hand, to identify those points corresponding to selfintersections of the curve. In performing all these tasks, once again, we follow the methodology of Corless et al. (2002).

\subsubsection{Computation of other points on critical lines}

Let $\chi=\left\{x_{1}<x_{2}<\cdots<x_{r}\right\}$ be the $x$-coordinates of all critical points. For each $x_{\ell} \in \chi$ there may be other noncritical points on $\mathcal{C}$ with the same $x$-coordinate. The parameter values for these points are the solutions of the equation $x(t)=x_{\ell}$. Thus we compute the companion matrix pencil corresponding to the polynomial $x(t)-x_{\ell}$ and the generalized eigenvalues. To obtain the desired generalized eigenvalues we discard those complex non-real and infinite generalized eigenvalues. Moreover, we must also discard the generalized eigenvalues computed in the previous step.

\subsubsection{Noncritical lines}

Consider the elements of $\chi$, and define

$$
a_{0}=x_{1}-2, \quad a_{\ell}=\left(x_{\ell}+x_{\ell+1}\right) / 2,1 \leq \ell \leq r-1, \quad a_{r}=x_{r}+2 .
$$

By using a similar procedure as before, for each $0 \leq \ell \leq r$, we compute all the points on $\mathcal{C}$ whose $x$-coordinates are equal to $a_{\ell}$. In this case, there are no previously known solutions.

\subsubsection{The edges of the graph}

Once we have computed the curve points on the critical and noncritical lines, to form up the graph representing the topology of $\mathcal{C}$ we need to determine how these points are connected by segments; starting at $P_{0}=\left(x\left(t_{0}\right), y\left(t_{0}\right)\right)$ a regular point on the curve lying on a noncritical line, we use a set of rules based on the sign of $x^{\prime}\left(t_{0}\right)$ to connect $P_{0}$ to the points on the critical lines on its left and right hand sides. The rules which we apply to complete the connecting of the points are stated in the next proposition. For a detailed proof of this result see Alcázar and Díaz-Toca (2010). 
Proposition 12. Let $x=a_{i}$ be a noncritical line. Suppose $x=x_{i}$ is the closest critical line on the left hand side (if $i>0$ ) and $x=x_{i+1}$ is the closest critical line on the right hand side (if $i<r$ ) of $x=a_{i}$. Let also $P_{0}=\left(x\left(t_{0}\right), y\left(t_{0}\right)\right)$ be a regular point of $\mathcal{C}$ lying on this noncritical line, that is $x\left(t_{0}\right)=a_{i}$. Let $\mathcal{V}_{i}$ be the set of real values of the parameter generating the points in $\mathcal{C} \cap\left\{x=x_{i}\right\}$. Then

1. If $x^{\prime}\left(t_{0}\right)>0$ then $P_{0}$ must be connected to the point in $\mathcal{C} \cap\left\{x=x_{i}\right\}$ generated by the biggest element of $\mathcal{V}_{i}$ which is smaller than $t_{0} . P_{0}$ must also be connected to the point in $\mathcal{C} \cap\left\{x=x_{i+1}\right\}$ generated by the smallest element of $\mathcal{V}_{i+1}$ which is bigger than $t_{0}$.

2. If $x^{\prime}\left(t_{0}\right)<0$ then $P_{0}$ must be connected to the point in $\mathcal{C} \cap\left\{x=x_{i}\right\}$ generated by the smallest element of $\mathcal{V}_{i}$ which is greater than $t_{0}$. Here again, $P_{0}$ must be connected to the point in $\mathcal{C} \cap\left\{x=x_{i+1}\right\}$ generated by the biggest element of $\mathcal{V}_{i+1}$ which is smaller than $t_{0}$.

This last step of the algorithm is crucial because it usually fails if numerical errors have been incurred during the process (see Section 6.3.1). In this case, we increase the computing precision and repeat the computation.

\subsection{The algorithm}

\section{Input:}

- The degree bounds $m$ and $n$ for $x(t)$ and $y(t)$ with respect to $t$, respectively.

- The parameter values $\boldsymbol{\tau}=\left(\tau_{1}<\ldots<\tau_{d+1}\right)$ and $\boldsymbol{\sigma}=\left(\sigma_{1}<\ldots<\sigma_{d-1}\right)$, and the corresponding values of points coordinates $\left\{\left(x\left(\tau_{i}\right), y\left(\tau_{i}\right)\right): 1 \leq i \leq d+1\right\}$ and $\left\{\left(x\left(\sigma_{i}\right), y\left(\sigma_{i}\right)\right): 1 \leq i \leq d-1\right\}$.

Output: The graph representing the topology of the parametric curve $(x(t), y(t))$.

1. Singular points

(a) For $k=1, \ldots, d-1$ compute the matrices $\mathbf{B}_{k}$ by using Formula (36).

(b) Construct the companion matrix pencil $\left(\mathbf{C}_{0}, \mathbf{C}_{1}\right)$ and compute the generalized eigenvalues. Discard those complex non-real and infinite eigenvalues.

2. Ramification points

(a) Compute the values $\left(x^{\prime}\left(\tau_{i}\right): 0 \leq i \leq m\right)$.

(b) Construct the corresponding matrix pencil $\left(\mathbf{C}_{0}, \mathbf{C}_{1}\right)$ associated with $\left(\tau_{i}: 1 \leq i \leq m\right)$ and $\left(x^{\prime}\left(\tau_{i}\right): 1 \leq\right.$ $i \leq m)$ and compute the generalized eigenvalues. Discard those complex non-real and infinite eigenvalues.

3. Coordinates of critical points

(a) Collect all the finite and real generalized eigenvalues obtained in the previous steps.

(b) Cluster all generalized eigenvalues corresponding to the same value of the parameter $t$.

(c) For each generalized eigenvalue $t$ obtained in this way, compute the $x$ and $y$ coordinates using the evaluation procedure for $x(t)$ and $y(t)$.

(d) To obtain the right number of singularities, separate the $x$-coordinates and points corresponding to self-intersections.

4. Other points on a critical line

Let $\left\{x_{\ell}: 1 \leq \ell \leq r\right\}$ be the set of the $x$-coordinates of critical points. Then for each $x_{\ell}$ we proceed as follows:

(a) Construct the matrix pencil associated with $\left(\tau_{i}: 1 \leq i \leq m+1\right)$ and $\left(x\left(\tau_{i}\right)-x_{\ell}: 1 \leq i \leq m+1\right)$ and compute the generalized eigenvalues. Discard all complex non-real and infinite eigenvalues in addition to the eigenvalues found in (3b).

(b) For each eigenvalue $t$, compute the $y$-coordinate using the evaluation procedure, and store the data $\left(t,\left(x_{\ell}, y(t)\right)\right)$ in a list.

5. Curve points on noncritical lines

Let $\left\{a_{0}<a_{1}<\cdots<a_{r}\right\}$ be the set defined by Equations (37). Then for each $\ell=0, \ldots, r$, we proceed as follows:

(a) Construct the companion matrix pencil associated with $\left(\tau_{i}: 1 \leq i \leq m+1\right)$ and $\left(x\left(\tau_{i}\right)-a_{\ell}: 1 \leq i \leq\right.$ $m+1$ ), and compute the generalized eigenvalues. Discard those complex non-real and infinite. 
(b) For each eigenvalue $t$, compute the $y$-coordinate using the evaluation procedure for $y(t)$, and store the data $\left(t,\left(a_{\ell}, y(t)\right)\right)$ in a list.

6. Edges of the graph

(a) Using Proposition 12, for each noncritical line and for each point $P_{0}$ on the noncritical line, determine the edges of the graph to the left hand side and to the right hand side. If this step fails, increase the precision and repeat the computation.

(b) Draw the graph using all the information collected from the previous steps.

Example 3. Here we present a detailed example showing the main steps of the algorithm.

Input:

$$
\begin{gathered}
m=6, \quad n=3, \quad \boldsymbol{\tau}=(-4,-3,-1,1,3,4,5), \quad \boldsymbol{\sigma}=(5,6,7,8,9), \\
\left\{\left(x\left(\tau_{i}\right), y\left(\tau_{i}\right)\right): 1 \leq i \leq 7\right\}=\left\{\left(\frac{16384}{3},-96\right),(845,-45),\left(-\frac{49}{3},-3\right),(-1 / 3,-1),(125,9),\left(\frac{4096}{3}, 32\right),\left(\frac{21175}{3}, 75\right)\right\}, \\
\left\{\left(x\left(\sigma_{i}\right), y\left(\sigma_{i}\right)\right):, i=1 \leq i \leq 5\right\}=\left\{\left(\frac{21175}{3}, 75\right),(25088,144),(70805,245),\left(\frac{512000}{3}, 384\right),(366597,567)\right\} .
\end{gathered}
$$

Singular points.

$$
\mathbf{B}(s)=\sum_{k=1}^{5} \mathbf{B}_{k} L_{k}(s ; \boldsymbol{\sigma}),
$$

with

$$
\begin{aligned}
\mathbf{B}_{1} & =\left(\begin{array}{ccccc}
\frac{171328}{9} & 12095 & \frac{60289}{9} & \frac{54283}{9} & 8573 \\
12095 & 7475 & 3795 & \frac{8785}{3} & 4395 \\
\frac{60289}{9} & 3795 & \frac{11683}{9} & \frac{2419}{9} & 1539 \\
\frac{54283}{9} & \frac{8785}{3} & \frac{2419}{9} & -\frac{4037}{9} & \frac{11449}{3} \\
8573 & 4395 & 1539 & \frac{11449}{3} & 19675
\end{array}\right), \mathbf{B}_{2}=\left(\begin{array}{cccccc}
32768 & 23432 & 14952 & \frac{38104}{3} & 15912 \\
23432 & 16263 & 9373 & \frac{20441}{3} & 8921 \\
14952 & 9373 & 3479 & \frac{2051}{3} & 3339 \\
\frac{38104}{3} & \frac{20441}{3} & \frac{2051}{3} & -\frac{9113}{9} & 7757 \\
15912 & 8921 & 3339 & 7757 & 35855
\end{array}\right), \\
\mathbf{B}_{3}= & \left(\begin{array}{ccccc}
\frac{524608}{9} & \frac{133819}{3} & \frac{270847}{9} & \frac{220093}{9} & \frac{84553}{3} \\
\frac{133819}{3} & \frac{98969}{3} & \frac{59777}{3} & \frac{124627}{9} & 16611 \\
\frac{270847}{9} & \frac{59777}{3} & \frac{68467}{9} & \frac{12907}{9} & \frac{19097}{3} \\
\frac{220093}{9} & \frac{124627}{9} & \frac{12907}{9} & -\frac{17797}{9} & \frac{126979}{9} \\
\frac{84553}{3} & 16611 & \frac{19097}{3} & \frac{126979}{9} & \frac{181825}{3}
\end{array}\right), \mathbf{B}_{4}=\left(\begin{array}{cccccc}
\frac{913408}{9} & 80616 & \frac{499816}{9} & \frac{392488}{9} & 47400 \\
80616 & 61711 & 37889 & \frac{76313}{3} & 28725 \\
\frac{499816}{9} & 37889 & \frac{131215}{9} & \frac{23941}{9} & 11075 \\
\frac{392488}{9} & \frac{76313}{3} & \frac{23941}{9} & -\frac{31457}{9} & \frac{71075}{3} \\
47400 & 28725 & 11075 & \frac{71075}{3} & 96575
\end{array}\right), \\
\mathbf{B}_{5}= & \left(\begin{array}{ccccc}
169792 & 137683 & 95429 & \frac{218821}{3} & 75929 \\
137683 & 107419 & 66203 & \frac{129769}{3} & 46739 \\
95429 & 66203 & 25459 & \frac{13577}{3} & 17995 \\
\frac{218821}{3} & \frac{129769}{3} & \frac{13577}{3} & -\frac{51677}{9} & \frac{112337}{3} \\
75929 & 46739 & 17995 & \frac{112337}{3} & 146755
\end{array}\right) .
\end{aligned}
$$

The corresponding companion matrix pencil is of dimension $30 \times 30$, and we compute its real generalized eigenvalues.

\section{Ramification points.}

By using Equation (16), we obtain the values of the derivatives at the nodes in $\tau_{i} \in \boldsymbol{\tau}$, for $1 \leq i \leq 6$.

$$
\left(x^{\prime}\left(\tau_{i}\right): 1 \leq i \leq 6\right)=\left(-\frac{75776}{9},-\frac{5902}{3}, \frac{322}{9}, \frac{14}{9}, \frac{1150}{3}, \frac{23552}{9}\right) .
$$


Since we need the values of the parameter $t$ such that $x^{\prime}(t)=0$, we compute the generalized eigenvalues of the companion matrix pencil of $x^{\prime}(t)$ in the Lagrange basis (see Definition 5),

$$
\left(\left(\begin{array}{ccccccc}
-4 & 0 & 0 & 0 & 0 & 0 & -\frac{75776}{9} \\
0 & -3 & 0 & 0 & 0 & 0 & -\frac{5902}{3} \\
0 & 0 & -1 & 0 & 0 & 0 & \frac{322}{9} \\
0 & 0 & 0 & 1 & 0 & 0 & \frac{14}{9} \\
0 & 0 & 0 & 0 & 3 & 0 & \frac{1150}{3} \\
0 & 0 & 0 & 0 & 0 & 4 & \frac{23552}{9} \\
-\frac{1}{7560} & \frac{1}{2688} & -\frac{1}{1440} & \frac{1}{960} & -\frac{1}{672} & \frac{1}{840} & 0
\end{array}\right),\left(\begin{array}{ccccccc}
1 & 0 & 0 & 0 & 0 & 0 & 0 \\
0 & 1 & 0 & 0 & 0 & 0 & 0 \\
0 & 0 & 1 & 0 & 0 & 0 & 0 \\
0 & 0 & 0 & 1 & 0 & 0 & 0 \\
0 & 0 & 0 & 0 & 1 & 0 & 0 \\
0 & 0 & 0 & 0 & 0 & 1 & 0 \\
0 & 0 & 0 & 0 & 0 & 0 & 0
\end{array}\right)\right) .
$$

Next, we compute the real generalized eigenvalues.

Coordinates of critical points.

After discarding all the infinite and complex non-real generalized eigenvalues computed in the previous steps and clustering them, we obtain the following real eigenvalues

$$
\{2,4 / 3,0,1.817849301,-1.557045304,0.6280848919\} \text {. }
$$

By evaluating $x(t)$ and $y(t)$ at these points we obtain

$$
\begin{gathered}
(x(2), y(2))=(x(0), y(0))=(0,0), \\
(x(4 / 3), y(4 / 3))=(0,-32 / 27), \\
(x(1.817849301), y(1.817849301))=(-0.5394877248,-0.6019308430), \\
(x(-1.557045304), y(-1.557045304))=(-31.91248931,-8.623665348), \\
(x(0.6280848919), y(0.6280848919))=(-0.7074367101,-0.5412076571) .
\end{gathered}
$$

Other points on critical lines.

Once the critical points are computed, we observe that the critical lines are:

$$
x=-31.91248931, x=-0.7074367101, x=-0.5394877248 \text { and } x=0 .
$$

For every critical line, we compute the corresponding companion matrix pencil and its generalized eigenvalues. We obtain that

- for $x=-31.91248931$, there are no more points on this line other than the one previously computed.

- for $x=-0.7074367101$, there are two more points generated by $t=-1.99599102166639009$ and $t=$ -.265397193898621986 . By evaluating $x(t)$ and $y(t)$ we obtain

$$
\begin{aligned}
& (x(-1.99599102166639009), y(-1.99599102166639009))=(-0.7074367101,-15.91994896), \\
& (x(-.265397193898621986), y(-.265397193898621986))=(-0.7074367101,-.1595647703) .
\end{aligned}
$$

- for $x=-0.5394877248$, there are four more points generated by $t=.403031516146586999, t=$ 0.860587628220808032 and $t=-.235702890233506990, t=-1.99694818957432996$. By evaluating $x(t)$ and $y(t)$ we obtain

$$
\begin{gathered}
(x(.403031516146586999), y(.403031516146586999))=(-0.5394877248,-.2594026221), \\
(x(0.860587628220808032), y(0.860587628220808032))=(-0.5394877248,-.8438614114), \\
(x(-.235702890233506990), y(-.235702890233506990))=(-0.5394877248,-.1242063799), \\
(x(-1.99694818957432996), y(-1.99694818957432996))=(-0.5394877248,-15.93903829) .
\end{gathered}
$$

- for $x=0$, there is one more point generated by $t=-2$. By evaluating $x(t)$ and $y(t)$ we obtain

$$
(x(-2), y(-2))=(0,-16) .
$$


Noncritical lines.

Following the same notation as above, let

$$
a_{0}=-32.91248931, a_{1}=-16.30996301, a_{2}=-.6234622175, a_{3}=-.2697438624, a_{4}=2 .
$$

Using a similar procedure as before, for each $0 \leq \ell \leq 4$, we find all points on $\mathcal{C}$ whose $x$-coordinates are equal to $a_{\ell}$. In this case, there are no previously known solutions.

Edges of the graph.

By Proposition 12, at this point, the only required computation is the evaluation of the derivatives at the points on the noncritical lines. To do such a computation we use Equation (16).

Output.

The graph of the curve can be found in Figure 7 where the plotted points correspond to the real points of the curve. From the more detailed graph on the right hand side we can observe that the curve is not in generic position.
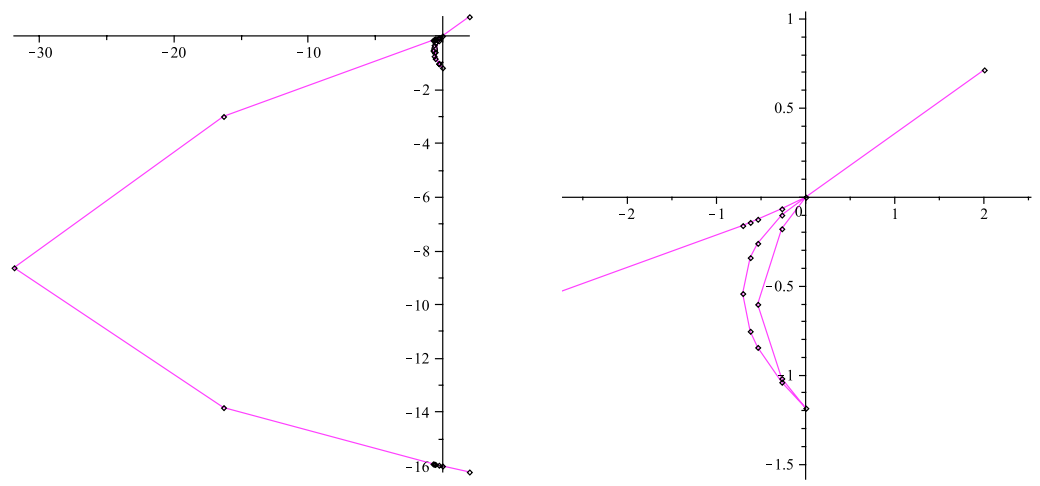

Figure 7: Graph of the Example 3

\subsection{Experimental results}

The following analysis can be used as an indication of the computational cost of the introduced method in this section to compute the topology of $(x(t), y(t))$. If the degrees of $x(t)$ and $y(t)$ with respect to $t$ are respectively bounded by $m$ and $n$, and $d=\max \{m, n\}$, then the algorithm computing the topology of the curve defined by $(x(t), y(t))$, presented in this section, requires $2 d$ point evaluations $(x(\tau), y(\tau))$, that is, $4 d$ single evaluations.

To obtain the $t$-values which generate singular points, we solve one generalized eigenvalue problem for a companion matrix pencil of size $d^{2}-d$. To determine the $t$-values which generate the ramification points, we solve one generalized eigenvalue problem for a companion matrix pencil of size $m+1$. To compute the coordinates of the non-critical points on every critical line, we solve $k$ generalized eigenvalue problems for companion matrices of size $m+2$ where

$$
k=(m-1)(n-1) \leq(d-1)^{2}
$$

is the number of critical lines ( $k$ agrees with the degree of $\mathrm{r}(s)$ ).

The described algorithm has been implemented in Maple. Table 3 presents some computational information about some of the examples we have analyzed (some of these examples have been taken from Gonzalez-Vega and Necula (2002)). Moreover, such examples have also been treated with the algorithm presented in Alcázar and Díaz-Toca (2010) in order to compare the timings. The information provided include:

- $m=\operatorname{deg}_{t} x(t)$ and $n=\operatorname{deg}_{t} y(t)$, 


\begin{tabular}{|c||c|c|c|c|c|c|c|c|}
\hline Ex. & $m$ & $n$ & precision & time $_{1}$ & time $_{2}$ & Total & precision $_{p}$ & time $_{p}$ \\
\hline \hline 1 & 3 & 3 & 10 & 0.015 & 0.016 & 0.140 & 10 & 0.089 \\
2 & 6 & 3 & 10 & 1.078 & 0.079 & 1.343 & 10 & 0.106 \\
3 & 6 & 4 & 10 & 1.687 & 0.094 & 2.063 & 10 & 0.128 \\
4 & 8 & 7 & 10 & 24.610 & 0.218 & 25.360 & 40 & 10.250 \\
5 & 4 & 5 & 10 & 0.219 & 0.078 & 0.719 & 10 & 0.135 \\
6 & 7 & 6 & 15 & 4.328 & 0.344 & 5.531 & 10 & 0.312 \\
7 & 10 & 10 & 20 & 51.063 & 0.597 & 52.719 & 70 & 0.626 \\
8 & 10 & 5 & 25 & 21.402 & 0.591 & 22.771 & 180 & 6.889 \\
9 & 10 & 4 & 60 & 32.351 & 0.924 & 34.977 & 400 & 174.350 \\
\hline
\end{tabular}

Table 3: Experimental results

- time $_{1}$ which indicates the computing time (in seconds) for determining the points on critical lines,

- time $_{2}$ which indicates the computing time for determining the points on noncritical lines,

- time $_{p}$ which indicates the computing time for the algorithm presented in Alcázar and Díaz-Toca (2010), and

- we have also included the number of digits used in the computations ("precision" for the algorithm described here and "precision $p$ " for the algorithm presented in Alcázar and Díaz-Toca (2010)).

One may observe that the costliest step is the computation of the points on the critical lines. The parametrizations corresponding to these examples are given in Appendix I.

The graphs corresponding to the examples in Table 3 are shown in Figure 8. From left to right we have Examples 1, 2, 3 in the first row, Examples 4, 5, 6 in the second row and Examples 7,8 and 9 in the third one.

\subsubsection{Possible errors}

We have mentioned that Step 6 usually detects the common numerical errors that lead to a wrong graph during the process:

- Whether there are more critical lines than supposed to be because the $x$-coordinates of critical points have been wrongly separated in Step 3(d) and one or more critical lines have been broken down in two.

- Whether there are less critical lines than supposed to be because the $x$-coordinates of critical points have been wrongly separated in Step 3(d) and one or more critical lines have not been computed. Here however, if such lines define the extremes of the graph, the error will not be detected in the last step.

- Whether the number of points on a critical line is less than supposed to be (Step 4).

- Whether the number of point on a non-critical point is greater than supposed to be (Step 5).

Let us say that even these errors are detected, we are working on how to avoid them (see Section 8 and Section 9).

\section{Using (but not computing) the implicit equation of $(x(t), y(t))$ to determine its topology}

In this section, we present a new approach for computing the topology of a curve specified by its parametrization $(x(t), y(t))$ in the Lagrange polynomial basis. The main algorithm to be used here is the same presented in Section 5 . We show how to evaluate $f(x, y)$ without explicitly computing it. These evaluations are essential to the idea of determining the topology of $f(x, y)=0$ without the necessity to compute it explicitly.

Let $\boldsymbol{\tau}=\left(\tau_{1}<\tau_{1}<\ldots<\tau_{m+n}\right)$ be a collection of distinct real numbers and suppose that we have the values $\left\{\left(x\left(\tau_{i}\right), y\left(\tau_{i}\right)\right): \tau_{i} \in \boldsymbol{\tau}\right\}$. We also assume that the curve is in generic position (see Definition 7 ). Furthermore, let $\boldsymbol{\sigma}=\left(\sigma_{1}<\sigma_{1}<\ldots<\sigma_{d+1}\right)$ be a collection of real numbers and $\mathbf{L}(x ; \boldsymbol{\sigma}) \in\left[\mathbb{P}_{d}\right]^{d+1}$ as described in Equation (11) be the Lagrange basis associated with $\boldsymbol{\sigma}$. We now show how to apply the methodology described in Section 5 under these conditions. 

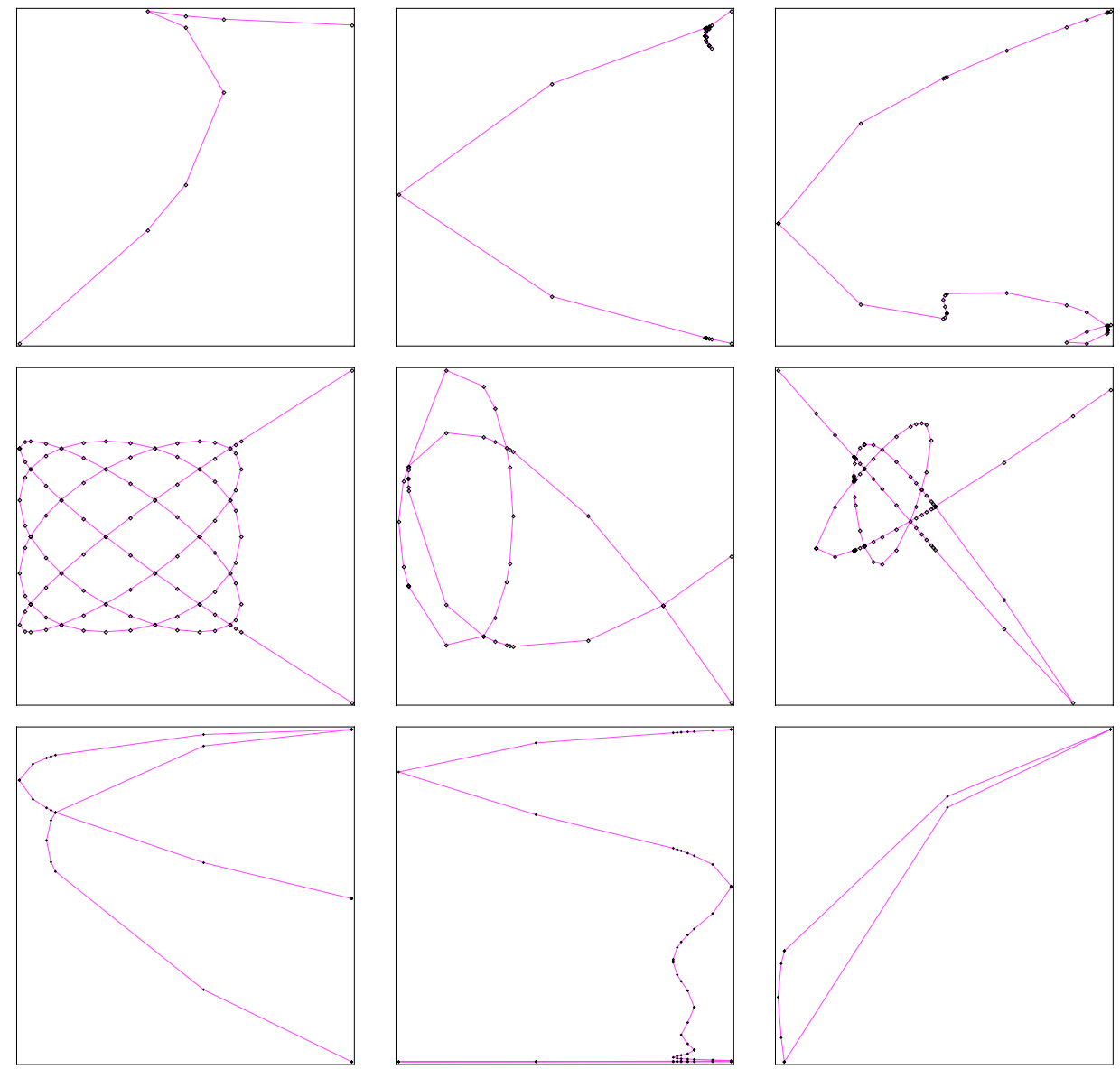

Figure 8: Parametric examples.

The implicit equation of the curve described above, $f(x, y)$, can be represented in the Lagrange basis in the following way:

$$
f(x, y)=\operatorname{resultant}_{t}(x(t)-x, y(t)-y)=\sum_{i=1, j=1}^{d+1} f_{i, j} L_{i}(x ; \sigma) L_{j}(y ; \sigma)
$$

where each $f_{i j}$ is the following concrete resultant

$$
f_{i j}=\operatorname{resultant}_{t}\left(x(t)-\sigma_{i}, y(t)-\sigma_{j}\right) .
$$

We may now proceed to compute these resultants as determinants. However, as computing determinants can be algebraically costly or numerically unstable, one of the goals here is to show how to avoid these computations. Instead, we use the following identity introduced in Apéry and Jouanolou (2006): given two polynomials $a(t)$ and $b(t)$ with $\operatorname{deg}(a)=n$ and $\operatorname{deg}(b)=m$, we have

$$
\operatorname{resultant}(a, b)=\sum_{I=U \amalg V,|U|=n,|V|=m} \frac{\prod_{u \in U} a(u) \prod_{v \in V} b(v)}{\prod_{u \in U, v \in V}(u-v)}
$$

with $I$ being a set of distinct $n+m$ nodes. 
By applying Equation (41) with $a(t)$ replaced by $x(t)-\sigma_{i}$ and $b(t)$ replaced by $y(t)-\sigma_{j}$, we obtain

$$
f_{i j}=\sum_{I=U \amalg V,|U|=n,|V|=m} \frac{\prod_{u \in U}\left(x(u)-\sigma_{i}\right) \prod_{v \in V}\left(y(v)-\sigma_{j}\right)}{\prod_{u \in U, v \in V}(u-v)}
$$

where $I=\boldsymbol{\tau}$. It is then possible to compute every $f_{i j}$ only by values.

Thus, the topology of the curve $(x(t), y(t))$ can be determined by computing the topology of $f(x, y)=0$ by using the algorithm presented in Section 5 (see 5.6). To apply such an algorithm we need to evaluate the partial derivative of $f(x, y)$ with respect to $y$. This will be achieved by using the formula:

$$
\frac{\partial f}{\partial y}(x, y)=\sum_{i=0, j=0}^{d} f_{i, j} L_{i}(x ; \sigma) \frac{\partial L_{j}(y, \sigma)}{\partial y} .
$$

Note that this method requires $m+n$ evaluations $(x(\tau), y(\tau))$, that is, $2(m+n)$ single evaluations.

Finally, we note that the graph obtained by following this approach can contain isolated points (with real coordinates) generated by complex, non-real values of the parameter $t$. They are real points on the curve $f(x, y)$ but they are not in the image of the parametrization $(x(t), y(t)$ ) when $t \in \mathbb{R}$ (see Fioravanti and Gonzalez-Vega $(2005))$.

Example 4. We consider the same curve as in Example 3; here, we do not intend to develop the example completely. We only show the main parts of the computation of the topology of a curve by using its implicit equation.

Input: Let

$$
m=6, \quad n=3, \quad \boldsymbol{\tau}=(-4,-3,-1,1,3,4,5,6,7), \quad \boldsymbol{\sigma}=(10,11,12,13,14,15,16,17)
$$

with

$$
\begin{gathered}
\left\{\left(x\left(\tau_{i}\right), y\left(\tau_{i}\right)\right): 1 \leq i \leq 9\right\}= \\
\left\{\left(\frac{16384}{3},-96\right),(845,-45),\left(-\frac{49}{3},-3\right),(-1 / 3,-1),(125,9),\left(\frac{4096}{3}, 32\right),\left(\frac{21175}{3}, 75\right),(25088,144),(70805,245)\right\} .
\end{gathered}
$$

Then, by using Equation (39), we can compute the $(d+1) \times(d+1)$ matrix $\left(f_{i, j}\right)$ of resultants

$$
f_{i j}=\operatorname{resultant}_{t}\left(x(t)-\sigma_{i}, y(t)-\sigma_{j}\right) \text {. }
$$

At the same time, we can compute these resultants by values by applying Equation (42). Hence we have

$$
\left(f_{i, j}\right)=\left(\begin{array}{ccccccccc}
\frac{1980353000}{729} & \frac{370968943}{81} & \frac{199146952}{27} & \frac{8336248727}{729} & & \frac{4171681400}{243} & \frac{676938125}{27} & \frac{26061267032}{729} \\
\frac{1944208001}{729} & \frac{365237048}{81} & \frac{196504303}{27} & \frac{8240097848}{729} & & \frac{4129431371}{243} & \frac{670865288}{27} & \frac{25852596677}{729} \\
\frac{1908581888}{729} & \frac{39952717}{9} & \frac{193888960}{27} & \frac{8144806541}{729} & \frac{4087511552}{243} & \frac{664834319}{27} & \frac{25645205312}{729} \\
\frac{1873470287}{729} & \frac{353980672}{81} & \frac{191300761}{27} & \frac{8050370432}{729} & \frac{4045920485}{243} & \frac{658845056}{27} & \frac{25439088563}{729} \\
\frac{1838868824}{729} & \frac{348455219}{81} & \frac{188739544}{27} & \frac{7956785147}{729} & \frac{4004656712}{243} & \frac{652897337}{27} & \frac{25234242056}{729} \\
\frac{1804773125}{729} & \frac{114332536}{27} & \frac{186205147}{27} & \frac{7864046312}{729} & \frac{3963718775}{243} & \frac{646991000}{27} & \frac{25030661417}{729} \\
\frac{1771178816}{729} & \frac{337607353}{81} & \frac{183697408}{27} & \frac{7772149553}{729} & \frac{3923105216}{243} & \frac{641125883}{27} & \frac{24828342272}{729}
\end{array}\right)
$$

From this matrix, we may compute the graph of the curve without the explicit computation of the implicit equation of such a curve. This is the matrix that can be used to evaluate the implicit equation of the curve according to Equation (39). 
The approach introduced here can be easily adapted to deal with the case of computing the topology of $(x(t), y(t))$ when $x(t)$ and $y(t)$ are rational functions and not polynomials. If

$$
x(t)=\frac{x_{n}(t)}{x_{d}(t)}, \quad y(t)=\frac{y_{n}(t)}{y_{d}(t)},
$$

to compute the topology of $(x(t), y(t))$ we replace $x(t)-x$ with $x_{n}(t)-x \cdot x_{d}(t)$ and $y(t)-y$ with $y_{n}(t)-y \cdot y_{d}(t)$ in Equations (39) and (40).

\section{Numerical Aspects}

In this section we make some short considerations on the numerical methods used in our algorithms.

It is known that the conversion between different polynomial bases can be unstable, and the instability increases with the degree (see, for example, Hermann (1996)). Hence, when the data is given by values, working directly in the Lagrange basis is usually better than working in other bases, even in the Bernstein one (see Berrut and Trefethen (2004); Higham (2004); Corless and Watt (2004); Corless (2006); Shakoori (2007)).

If for a polynomial $p(t)$ of degree $d$, one uses the Lagrange interpolation formula usually presented in textbooks

$$
p(t)=\sum_{i=1}^{d+1} p_{i} L_{i}(t ; \boldsymbol{\tau}),
$$

each evaluation of $p(t)$ requires $O\left(d^{2}\right)$ additions and multiplications. On the contrary, if one uses the barycentric form (10), the calculation of the barycentric weights (8), which are independent of $t$, require $O\left(d^{2}\right)$ flops, but they are done only once. The remaining operations for each evaluation of $p(x)$ require $O(d)$ flops only, once the numbers $\omega_{i}$ are known. In addition, it is known that the evaluation using the barycentric form is numerically more stable (see Berrut and Trefethen (2004)).

\subsection{Methods for computing generalized eigenvalues}

Without a doubt, the crucial step in the presented algorithms is the computation of generalized eigenvalues and for this task, we have used the existing routine in Maple which is quite successful in most cases. The stability of the rootfinding problem for univariate polynomials by way of standard methods to compute eigenvalues of companion matrices has been studied for the monomial basis (see Edelman and Murakami (1995); Toh and Trefethen (1994)), and for the Bernstein basis (see Jónsson and Vavasis (2004)). For the bivariate case (expressed in the monomial basis), in Jónsson and Vavasis (2005), the authors propose the use of the Macaulay resultant and analyze the error due to roundoff of the $Q Z$ method for computing generalized eigenvalues. However we have not found in the literature studies of stability of the $Q Z$ method for the matrix pencil in the Lagrange basis. The $Q Z$ method requires $O\left(d^{2}\right)$ in storage and $O\left(d^{3}\right)$ flops for the arithmetic. However, for our particular structure of the matrix pencil, the storage cost should be $O(d)$ and the arithmetic cost $O\left(d^{2}\right)$, (see Corless (2006)).

In Amiraslani (2006); Amiraslani et al. (2006) the authors have presented two variations of the Rayleigh quotient iteration method $(R Q I)$ for generalized eigenvalue problems, called constrained and unconstrained. Suppose that the matrices $\mathbf{C}_{0}$ and $\mathbf{C}_{1}$ are of size $r(d+2) \times r(d+2)$ as in (21). With both methods they have $O\left(d r^{2}\right)$ storage cost and $O\left(d^{2} r^{2}+r^{3}\right)$ arithmetic cost. For the constrained method the initial guesses need to be near the convex hull of the interpolation nodes, while the unconstrained method shows convergence almost everywhere.

Bini, Gemignani and Pan (Bini et al. (2005)) implemented a fast and numerically stable $Q R$-based algorithm for computing all eigenvalues of a generalized companion matrix, for a class of structured matrices including the kind of companion matrix pencils of a polynomial $(r=1)$ considered in the present paper. The $Q R$ iteration preserves the structure of the matrices. Each iteration requires $O(d)$ arithmetic operations, using $O(d)$ memory storage. The method could probably be extended to the computation of eigenvalues of a matrix polynomial. 


\subsection{Scaling and clustering}

In Corless (2006), the author extends the results of Corless and Watt (2004) to matrix polynomials expressed in the Lagrange basis, and investigates the effect of the geometry of the nodes on the conditioning of the problem. As in the monomial basis, he points out the importance of the scaling operation. The generalized eigenvalues remain unchanged if in (21) all the blocks $\mathbf{B}_{i}$ are multiplied by any nonsingular matrix. The last $r$ rows of $\mathbf{C}_{0}$ can also be multiplied by any nonsingular matrix without changing the generalized eigenvalues. It is usually advisable to do a suitable scaling to improve accuracy. However, we scaled the sample matrices and the nodes on examples which needed more precision, and we did not appreciate much improvement.

Another issue mentioned in Corless (2006) is the possibility of roots at infinity caused by oversampling. Our algorithms clearly present such a problem and we are studying the use of the dynamic evaluation to solve it.

After eliminating the infinite eigenvalues, it is common to find certain clusters of eigenvalues. The generalized eigenvalues in one of those clusters come from the same root of the determinant of the Bézout matrix. In the complex plane, they appear in a cross or star-shaped distribution, close to each other, with a real point at the center, and the whole cluster is replaced by this real value.

\subsection{Conditioning and faraway roots}

Conditioning of a problem measures the sensitivity of the solution to perturbations of the data. To decide whether a basis is well-conditioned or ill-conditioned one needs estimates of some condition number. Dividing an evaluation condition number by the norm of the derivative gives a rootfinding condition number. For the evaluation of a function, we can use one of the following condition numbers (see Lyche and Peña (2004)). Let $\mathcal{U}$ be a finite-dimensional vector space of functions defined on $\Omega \subset \mathbb{R}^{n}$, and let $\boldsymbol{\Phi}=\left(\phi_{0}, \ldots, \phi_{d}\right)$ be a basis for $\mathcal{U}$. Given $f=\sum_{i=0}^{d} c_{i} \phi_{i}$ we consider a perturbation $\delta=\left(\delta_{i}: 0 \leq i \leq d\right)$ of the coefficients $c_{i}$, producing $g=\sum_{i=0}^{d}\left(1+\delta_{i}\right) c_{i} \phi_{i}$. Then, if $x \in \Omega$,

$$
|f(x)-g(x)|=\left|\sum_{i=0}^{d} \delta_{i} c_{i} \phi_{i}(x)\right| \leq\|\delta\|_{\infty} \sum_{i=0}^{d}\left|c_{i} \phi_{i}(x)\right| .
$$

A condition number for the evaluation of $f$ at $x$ using the basis $\boldsymbol{\Phi}$ is

$$
C_{\boldsymbol{\Phi}}(f, x)=\sum_{i=0}^{d}\left|c_{i} \phi_{i}(x)\right| .
$$

Taking into account the size of $f$ we get the following condition numbers

$$
\begin{aligned}
\operatorname{cond}(\boldsymbol{\Phi} ; f, x) & =\frac{C_{\boldsymbol{\Phi}}(f, x)}{\|f\|_{\infty}}=\frac{\sum_{i=0}^{d}\left|c_{i} \phi_{i}(x)\right|}{\left\|\sum_{i=0}^{d} c_{i} \phi_{i}\right\|_{\infty}}, \\
\operatorname{cond}(\boldsymbol{\Phi} ; f) & =\sup _{x \in \Omega} \operatorname{cond}(\boldsymbol{\Phi} ; f, x) \\
\operatorname{cond}(\mathbf{\Phi}) & =\sup _{f \in \mathcal{U}} \operatorname{cond}(\boldsymbol{\Phi} ; f) .
\end{aligned}
$$

Therefore, for $\epsilon=\|\delta\|_{\infty}$,

$$
\begin{aligned}
|f(x)-g(x)| & \leq \epsilon C_{\boldsymbol{\Phi}}(f, x), \\
\frac{|f(x)-g(x)|}{\|f\|_{\infty}} & \leq \epsilon \operatorname{cond}(\boldsymbol{\Phi} ; f, x) \leq \epsilon \operatorname{cond}(\boldsymbol{\Phi} ; f) \leq \epsilon \operatorname{cond}(\boldsymbol{\Phi}) .
\end{aligned}
$$

This condition numbers can be used for polynomials represented in the Lagrange basis.

The importance of the geometry is remarked in Corless (2006). The roots inside a region defined by the interpolation nodes tend to be well-conditioned, whilst roots outside this region can be badly conditioned. To take advantage of this observation, when a high precision is needed, we should redefine the nodes inside the region defined by the computed solutions and repeat the computations. 


\section{Conclusions and future work}

We have presented several approaches for computing the topology of a given real algebraic plane curve described by its values in the Lagrange polynomial basis. The first algorithm is designed for problems in which the given data corresponds to an implicitly defined algebraic curve. The second algorithm however, is designed to be used for parametric curves. In both cases, the defining polynomial equations are specified only by their values at some sample points. Moreover, the degrees are known, can be deduced or are upper bounded. The key idea is to find the roots of the determinant of a particular Bézout matrix without computing the determinant. This is achieved by computing the generalized eigenvalues of a companion matrix pencil associated with the Bézout matrix. If the precision and other numerical parameters are adjusted appropriately, the algorithms presented here are very accurate. They are also efficient, always in the parametric case, and for moderate degrees in the implicit case. The size of the companion matrix pencil could be quite big in the implicit case, producing an increase in the computational cost.

One of our next goals is to reduce the computational time by using better methods for computing the generalized eigenvalues. These methods should take full advantage of the particular structure of the companion matrix pencil. Using such a particular structure, we also expect to find a practical bound which allows us to confidently discard large generalized eigenvalues as infinite ones. The desired bound for generalized eigenvalues may follow from Hadamard's inequality for determinants when applied to the companion matrix pencil in the Lagrange basis (see Corless et al. (2002) for the monomial case).

In addition, it would be interesting to study which properties of curves (normality, for instance) can be determined by values.

\section{Acknowledgements}

The authors thank the referees for the good suggestions that have helped to improve the paper.

The third and fourth authors are partially supported by the Spanish MEC grants MTM2008-04699-C0303/MTM and MTM2011-25816-C02-02. The sixth author is supported by the SAGA network.

\section{References}

Abhyankar, S., 1990. Algebraic geometry for scientists and engineers. In: Mathematical Surveys and Monographs. A.M.S.

Alcázar, J., Díaz-Toca, G., 2010. Topology of 2d and 3d rational curves. Computer Aided Geometric Design 27, $483-502$.

Amiraslani, A., May 2006. Algorithms for matrices, polynomials, and matrix polynomials. Ph.D. thesis, University of Western Ontario, London, Canada.

Amiraslani, A., Aruliah, D. A., Corless, R. M., 2006. The Rayleigh quotient iteration for generalized companion matrix pencils. Preprint.

Amiraslani, A., Corless, R. M., Lancaster, P., 2009. Linearization of matrix polynomials expressed in polynomial bases. IMA Journal of Numerical Analysis 29 (1), 141-157.

Apéry, F., Jouanolou, J. P., 2006. Résultant et sous-résultants : le cas d'une variable : avec exercices corrigés,. Hermann,, Paris.

Arnon, D., McCallum, S., 1988. A polynomial time algorithm for the topological type of a real algebraic curve. Journal of Symbolic Computation 5, 213-236.

Bajaj, C., Hoffmann, C. M., Lynch, R. E., Hopcroft, J. E. H., 1988. Tracing surface intersections. Computer Aided Geometric Design 5, 285-307.

Barnett, S., 1989. A Bezoutian matrix for Chebyshev polynomials. In: Application of Matrix Theory. Vol. 22. The Clarendon Press, New York, pp. 137-149. 
Barnett, S., 1990. Matrices: Methods and Applications. Clarendon, Oxford.

Berrut, J., Trefethen, L., 2004. Barycentric Lagrange interpolation. SIAM Review 46 (3), 501-517.

Bini, D., Gemignani, L., Pan, V., 2005. Fast and stable qr eigenvalue algorithms for generalized companion matrices and secular equations. Numer. Math. 100 (3), 373-408.

Bini, D., Pan, V., 1994. Polynomial and matrix computations. fundamental algorithms. Progress in Theoretical Computer Science 1.

Bini, D. A., Gemignani, L., 2004. Bernstein-Bezoutian matrices. Theoretical Computer Science 315 (2-3), 319333.

Busé, L., Khalil, H., Mourrain., B., 2005. Resultant-Based Method for Plane Curves Intersection Problems. No. 3718. pp. 75-92.

Cellini, P., Gianni, P., Traverso, C., 1991. Algorithms for the shape of semialgebraic sets: a new approach. Lecture Notes in Computer Sci. 539, 1-18.

Cheng, J., Lazard, S., Peñaranda, L., Pouget, M., Rouillier, F., Tsigaridas, E., 2010. On the topology of real algebraic plane curves. Mathematics and Computer Science Volume 4 (1), 113-137.

Corless, R. M., June 2004. Generalized companion matrices in the Lagrange basis. In: Gonzalez-Vega, L., Recio, T. (Eds.), Proceedings EACA. pp. 317-322.

Corless, R. M., 2006. Symbolic-Numeric Computation. Birkhauser, Ch. On a Generalized Companion Matrix Pencil for Matrix Polynomials Expressed in the Lagrange basis, pp. 1-18.

Corless, R. M., Gonzalez-Vega, L., Necula, I., Shakoori, A., 2002. Topology determination of implicitly defined real algebraic plane curves. Third International Workshop, Timisoara, Romania.

Corless, R. M., Rezvani, N., Amiraslani, A., 2007. Pseudospectra of matrix polynomials that are expressed in alternative bases. Mathematics in Computer Science.

Corless, R. M., Watt, S. M., September 2004. Bernstein bases are optimal, but, sometimes, Lagrange bases are better. In: Proceedings of SYNASC, Timisoara. MIRTON Press, pp. 141-153.

Cucker, F., Gonzalez-Vega, L., Rosello, F., 1991. On algorithms for real algebraic plane curves. Progress in Mathematics, Birkhausser 94, 63-88.

Demmel, J. W., 1997. Applied Numerical Linear Algebra. Society for Industrial and Applied Mathematics, Philadelphia.

Diaz-Toca, G. M., Fioravanti, M., Gonzalez-Vega, L., Shakoori, A., 2012. Using implicit equations of parametric curves and surfaces without computing them: polynomial algebra by valuesPreprint.

Diaz-Toca, G. M., Gonzalez-Vega, L., 2002. Barnett's theorems about the greatest common divisor of several univariate polynomials through bezout-like matrices. Journal of Symbolic Computation 34, 59-81.

Edelman, A., Murakami, H., April 1995. Polynomial roots from companion matrix eigenvalues. Mathematics of Computation 64 (210), 763-776.

Eigenwillig, A., Kerber, M., Wolpert, N., July 2007. Fast and exact geometric analysis of real algebraic plane curves. In: Proceedings ISSAC'07.

Farouki, R. T., Han, C. Y., Hass, J., 2005. Boundary evaluation algorithms for minkowski combinations of complex sets using topological analysis of implicit curves numerical algorithms. Numerical Algorithms 40 (3), 251-283.

Feng, H., 1992. Decomposition and computation of the topology of plane real algebraic curves. Ph.D. thesis, The Royal Institute of Technology, Stockholm, Sweden. 
Fioravanti, M., Gonzalez-Vega, L., 2005. On the geometric extraneous components appearing when using implicitization. In: Mathematical Methods for Curves and Surfaces. Nashboro Press, pp. 157-168.

Gianni, P., Traverso, C., 1983. Shape determination of real curves and surfaces. Ann. Univ. Ferrara Sez VII Sec. Math XXIX,, 87-109.

Gohberg, I. G., Lancaster, P., Rodman., L., 1982. Matrix polynomials. Academic Press.

Gonzalez-Vega, L., El Kahoui, M., 1996. An improved upper complexity bound for the topology computation of a real algebraic plane curve. Journal of Complexity 12, 527-544.

Gonzalez-Vega, L., Necula, I., 2002. Efficient topology determination of implicitly defined algebraic plane curves. Computer Aided Geometric Design 19 (9), 719-743.

Hass, J., Farouki, R. T., Han, C. Y., Song, X., Sederberg, T. W., 2007. Guaranteed consistency of surface intersections and trimmed surfaces using a coupled topology resolution and domain decomposition scheme. Advances in Computational Mathematics 27 (1), 1-26.

Heinig, G., Rost, K., 1984. Algebraic methods for toeplitz-like matrices and operators. Operator Theory: Advances and Applications 13.

Helmke, U., Fuhrmann, P. A., 1989. Bezoutians. Linear Algebra and Its Applications 122/123/124, 1039-1097.

Hermann, T., 1996. On the stability of polynomial transformations between Taylor, Bézier, and Hermite forms. Numerical Algorithms 13, 307-320.

Higham, N. J., 2004. The numerical stability of barycentric Lagrange interpolation. IMA Journal of Numerical Analysis 24, 547-556.

Hong, H., 1996. An efficient method for analyzing the topology of plane real algebraic curves. Mathematics and Computers in Simulation, $42(4-6), 571-582$.

Jónsson, G. F., Vavasis, S., 2004. Solving polynomials with small leading coefficients. Siam Journal on Matrix Analysis and Applications 26 (2), 400-414.

Jónsson, G. F., Vavasis, S. A., 2005. Accurate solution of polynomial equations using macaulay resultant matrices. Math. Comput. 74 (249), 221-262.

Kerber, M., 2009. Geometric algorithms for algebraic curves and surfaces. Ph.D. thesis, Saarland University.

Keyser, J., Culver, T., Manocha, D., Krishnan, S., 2000. Efficient and exact manipulation of algebraic points and curves. Computer Aided Design, 32 (11), 694-662.

Labs, O., 2010. A list of challenges for real algebraic plane curve visualization software. In: Nonlinear Computational Geometry, I.Z.Emiris et al. (eds.). Vol. 151 of The IMA Volumes in Mathematics and its Applications. Springer, pp. 137-164.

Lionel, A., Mourrain, B., Wintz, J., 2008. Topology and arrangement computation of semi-algebraic planar curves. Computer Aided Geometric Design 25 (8), 631-651.

Lyche, T., Peña, J., 2004. Optimally stable multivariate bases. Advances in Computational Mathematics 20, 149-159.

Manocha, D., Demmel, J. W., 1995. Algorithms for intersecting parametric and algebraic curves II; multiple intersections. Computer Vision, Graphics and Image Processing: Graphical Models and Image Processing $57(2), 81-100$.

Necula, I., 2005. Manipulación de curvas y superficies en CAGD mediante técnicas simbólico-numéricas. Ph.D. thesis, Universidad de Cantabria, Universidad de Cantabria, Santander, Cantabria, Spain. 
Pérez-Díaz, S., 2007. Computation of the singularities of parametric plane curves. Journal of Symbolic Computation, 835-857.

Roy, M.-F., 1996. Basic algorithms in real algebraic geometry and their complexity: from sturm's theorem to the existential theory of reals. Lectures in Real Geometry, de Gruyter Exp. Math., de Gruyter 23, 1-67.

Roy, M.-F., Szpirglas, A., 1990. Complexity of the computation of cylindrical decomposition and topology of real algebraic curves using thom's lemma. Lecture Notes in Mathematics 1420, 223-236.

Sakkalis, T., 1991. The topological configuration of a real algebraic curve. Bulletin Australian Mathematical Society $43(1), 37-50$.

Schneider, C., Werner, W., 1984. Polynomial interpolation: Lagrange versus Newton. Mathematics of Computation 43 (167), 205-217.

Seidel, R., Wolpert, N., 2005. On the exact computation of the topology of real algebraic curves (exploiting a little more geometry and a little less algebra). In: Proceedings of the 21-st Annual ACM Symposium on Computational Geometry. pp. 107-115.

Shakoori, A., 2007. Bivariate polynomial solver by values. Ph.D. thesis, The University of Western Ontario.

Song, X., Sederberg, T. W., Zheng, J., Farouki, R. T., Hass, J., 2004. Linear perturbation methods for topologically consistent representations of free-form surface intersections computer aided geometric design. Computer Aided Geometric Design 21 (3), 303-319.

Tisseur, F., Higham, N. J., 2001. Structured pseudospectra for polynomial eigenvalue problems, with applications. SIAM J. Matrix Anal. Appl. 23 (1), 187-208.

Toh, K.-C., Trefethen, L. N., 1994. Pseudozeros of polynomials and pseudospectra of companion matrices. Numerische Mathematik 68, 403-425.

Trefethen, L. N., 2000. Spectral Methods in Matlab. SIAM.

van den Essen, A., Yu, J.-T., 1997. The d-resultant, singularities and the degree of unfaithfulness. Proc. Amer. Math. Soc. 125 (3), 689-695.

\section{Appendix I}

Example 1: $\varphi(t)=\left(t^{3}+2 t^{2}, t^{3}-2 t^{2}\right)$.

Example 2: $\varphi(t)=\left(t^{6}-\frac{8}{3} t^{5}-\frac{20}{9} t^{4}+\frac{32}{3} t^{3}-\frac{64}{9} t^{2}, t^{3}-2 t^{2}\right)$.

Example 3: $\varphi(t)=\left(5 t^{6}-18 t^{5}-15 t^{4}+80 t^{3}-120 t, 3 t^{4}-4 t^{3}-12 t^{2}+12 t\right)$.

Example 4: $\varphi(t)=\left(t^{8}-8 t^{6}+20 t^{4}-16 t^{2}+2, t^{7}-7 t^{5}+14 t^{3}-7 t\right)$.

Example 5: $\varphi(t)=\left(\frac{1}{4} t^{4}-\frac{7}{6} t^{3}+\frac{1}{4} t^{2}+\frac{8}{3} t, \frac{1}{15} t^{5}-\frac{5}{12} t^{4}+\frac{1}{6} t^{3}+\frac{23}{12} t^{2}-\frac{11}{15} t-1\right)$.

Example 6: $\varphi(t)=\left(\frac{43}{10080} t^{7}-\frac{1}{480} t^{6}-\frac{101}{1440} t^{5}+\frac{1}{96} t^{4}-\frac{13}{180} t^{3}+\frac{59}{120} t^{2}+\frac{172}{105} t, \frac{11}{840} t^{6}-\frac{9}{560} t^{5}-\frac{107}{336} t^{4}\right.$

$$
\left.+\frac{103}{336} t^{3}+\frac{1011}{560} t^{2}-\frac{83}{105} t-1\right)
$$

Example 7: $\varphi(t)=\left(t^{10}-38 t^{8}-80 t^{6}-66 t^{5}+43 t^{4}-66 t^{2}-35 t,-22 t^{10}+49 t^{9}+96 t^{6}+57 t^{4}+99 t^{2}+85 t\right)$.

Example 8: $\varphi(t)=\left(t^{10}-55 t^{9}+1320 t^{8}-18150 t^{7}+157773 t^{6}-902055 t^{5}+3416930 t^{4}-8409500 t^{3}+12753576 t^{2}\right.$

$$
\left.-10628640 t+3628800, t^{5}+\frac{15}{2} t^{4}+\frac{85}{4} t^{3}+\frac{225}{8} t^{2}+\frac{137}{8} t+\frac{15}{4}\right) .
$$

Example 9: $\varphi(t)=$

$$
\left(t^{10}+800 t^{9}-2 t^{8}+\frac{100}{3} t^{7}-\frac{61}{7} t^{6}-888 t^{5}-9 t^{4}+95 t^{3}+11 t^{2}-488 t-407, t^{4}+400 t^{3}-831 t^{2}+91 t+68\right) .
$$

